\title{
Mixed Stabilized Finite Element Methods in Nonlinear Solid Mechanics. Part III: Compressible and incompressible plasticity
}

\author{
M. Cervera, M. Chiumenti, L. Benedetti and R. Codina \\ International Center for Numerical Methods in Engineering (CIMNE) \\ Technical University of Catalonia (UPC) \\ Edificio C1, Campus Norte, Jordi Girona 1-3, 08034 Barcelona, Spain.
}

KEYWORDS: mixed finite elements, stabilization, plasticity, strain softening, strain localization, mesh dependence.

\begin{abstract}
This paper presents the application of a stabilized mixed strain/displacement finite element formulation for the solution of nonlinear solid mechanics problems involving compressible and incompressible plasticity. The variational multiscale stabilization introduced allows the use of equal order interpolations in a consistent way. Such formulation presents two advantages when compared to the standard, displacement based, irreducible formulation: (a) it provides enhanced rate of convergence for the strain (and stress) field and (b) it is able to deal with incompressible situations. The first advantage also applies to the comparison with the mixed pressure/displacement formulation. The paper investigates the effect of the improved strain and stress fields in problems involving strain softening and localization leading to failure, using low order finite elements with continuous strain and displacement fields $(P 1 P 1$ triangles or tetrahedra and $Q 1 Q 1$ quadrilaterals, hexahedra, and triangular prisms) in conjunction with an associative frictional Drucker-Prager plastic model. The performance of the strain/displacement formulation under compressible and nearly incompressible deformation patterns is assessed and compared to a previously proposed pressure/displacement formulation. Benchmark numerical examples show the capacity of the mixed formulation to predict correctly failure mechanisms with localized patterns of strain, virtually free from any dependence of the mesh directional bias. No auxiliary crack tracking technique is necessary.
\end{abstract}




\section{Introduction}

In previous works $[1,2]$, the authors have formulated stable mixed stress/displacement and strain/ displacement finite elements with equal order interpolation for the solution of nonlinear problems in solid mechanics. The proposed formulation uses the sub-grid scale approach to circumvent the restrictiveness of the inf-sup compatibility conditions on the choice of the interpolation spaces. The objective of such formulation is to achieve a discrete scheme with enhanced stress accuracy. This means that the mixed formulation displays a global rate of convergence on stresses higher than the corresponding irreducible formulation. Such improvement of the convergence estimates also applies at local level. And this characteristic proves to be crucial in strain localization problems involving softening materials.

Strain localization inevitably occurs in softening materials subjected to monotonic straining. Once the peak stress is reached, and upon continuing straining, the stress decreases and strains concentrate inside a narrow band of material while the material outside the band unloads elastically. As the localization progresses, the width of the localization band diminishes and, unless there is a microstructural limitation, it tends to zero. The particular components of the strain tensor that localize during this process depend on the specific constitutive behavior of the material. In Rankine-type materials, only normal elongations localize, eventually forming tensile cracks; if the nonlinear behavior is incompressible, shear strains concentrate, leading to slip surfaces.

Quasi-singular strain or stress states occur at the vicinity of the propagating cracks or slip lines.

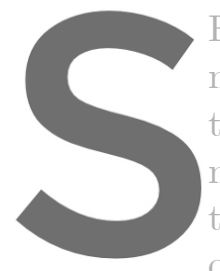
For linear elements an mulation fails to provice the tip of a notch or a mesh bias dependence tutive models. Contras of convergence for locals stress convergence. This th
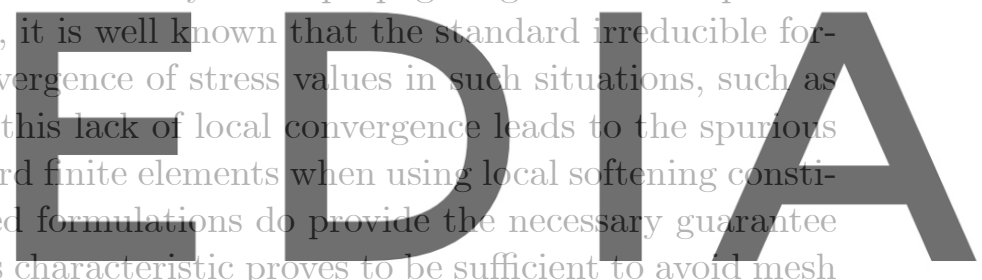
bias dependence of the numerically computed failure mechanisms and responses.

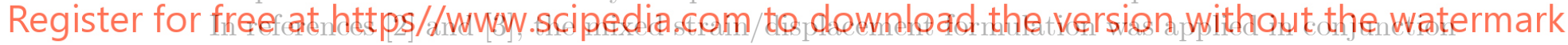

with an isotropic Rankine damage model, formulated in secant form, to model problems of tensile cracking propagation and failure. It was observed there that: (a) the resulting discrete FE model is well posed and stable, (b) the formulation is convergent and, on mesh refinement, it approaches the original continuum problem, and (c) the results obtained are not spuriously dependent of the finite element mesh used; they depend only on the actual material model (damage criterion in this case) adopted. This represented a significant advancement in the solution of such problems, particularly considering two noteworthy features of the approach. On one hand, it is of general application, in 2D and 3D problems, to structured and unstructured meshes and to simplicial or non simplicial elements. On the other hand, no "ad hoc" auxiliary crack tracking technique is necessary. However, the application of the proposed formulation to problems involving local softening plasticity models remained open.

In previous works, the authors have applied stabilized mixed displacement-pressure methods $([4,5,6,7,8]$ and $[9])$ to the solution of $J_{2}$ elasto-plastic problems with simplicial elements. In $J_{2}$ dependent problems, the plastic flow is isochoric and the main challenge for the discrete formulation is the incompressibility constraint. Unless this is properly dealt with, spurious pressure oscillations appear and the discrete solution is totally polluted. A stabilized mixed formulation 
provides a discrete problem which is fully stable, even for problems involving localization of shear strains and the formation of slip lines. The results obtained, both in terms of collapse mechanism and global load-deflection response, compare very favorably with those obtained with the standard irreducible formulation, which almost inevitably shows an unacceptable mesh dependence. Nevertheless, regarding the computation of the deviatoric stresses, the stabilized mixed pressuredisplacement formulation has the same convergence behavior than the irreducible formulation. This is because, in both formulations, the discrete deviatoric strains are computed by direct differentiation of the discrete displacement field. This means that the corresponding convergence rate is necessarily one order less than that of the displacements. When using linear interpolation for the displacements and in quasi-singular situations, this may prove to be insufficient. The remedy is to use an independent interpolation, linear at least, not only for the volumetric part of the strain (or stress) tensor, but for all of its components.

Therefore, the objectives of this paper are five: (1) to extend the stabilized mixed strain/displacement formulation to plasticity problems, (2) to investigate the effect of the improved strain and stress fields in problems involving strain softening and localization leading to failure, (3) to assess the performance of the formulation under nearly incompressible deformation patterns, (4) to compare the performance of the proposed formulation with the previously proposed pressure/displacement formulation and (5) to show that the formulation is applicable in $2 \mathrm{D}$ and 3D, to structured or unstructured meshes of triangles, quadrilaterals, tetrahedra, hexahedra or prisms. Both pressure sensitive and incompressible plasticity models are contemplated. To achieve this, the DruckerPrager plasticity model through the friction al null friction is assume

Inelastic plastic flow plasticity, it occurs in th
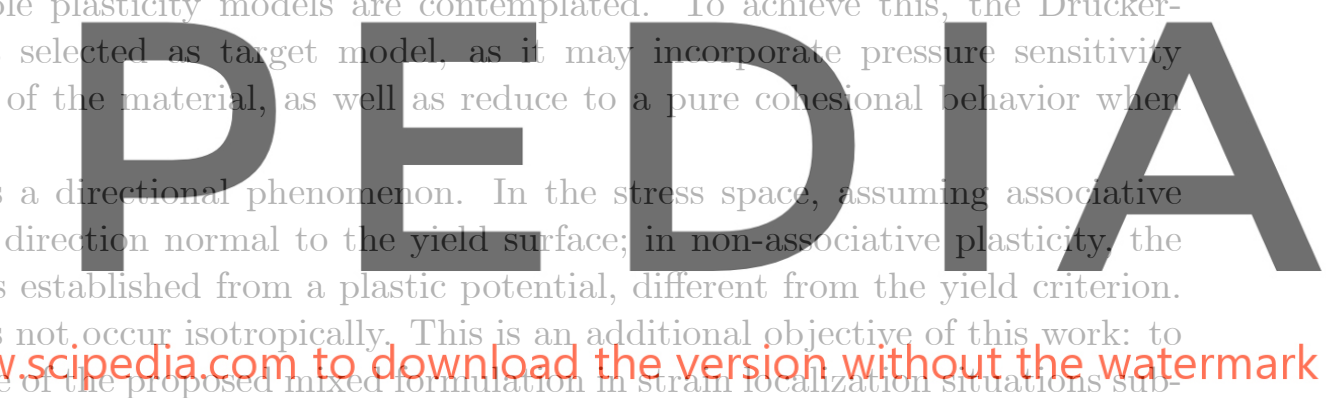

In any case, plasticity does not.occur isotropically. This is an additional objective of this work: to

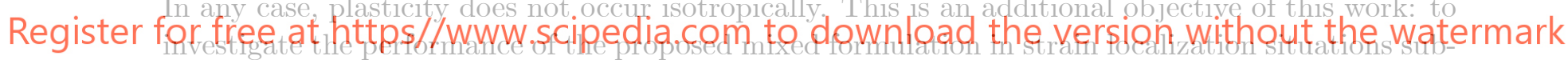

stantially different to those studied in previous works. Satisfactory performance under directional inelastic behavior, without spurious stress locking and without the need of auxiliary discontinuity tracking procedures, would reopen the path to the use of orthogonal and anisotropic constitutive models than cannot be used today in practical applications.

The outline of the paper is as follows. In Section 2, the stabilized mixed strain/displacement formulation for the solution of nonlinear solid mechanics problems is applied in conjunction with a small strain plasticity model. The continuum problem and the corresponding discrete formulation are introduced. Following the ideas in [1] and [2], stabilization of the latter is achieved by considering a residual-based subscale approach. Both algebraic and orthogonal subgrid scales are considered. Section 3 describes the implementation details for both stabilization procedures. Section 4 describes the Drucker-Prager plasticity model. Details on the return mapping, consistent tangent constitutive tensor and the consideration of the singular case of the apex of the yield surface are discussed. Section 5 presents selected numerical examples involving unstructured and structured low order finite elements meshes (triangles in 2D and triangular prisms in 3D) with continuous linear strain and displacement fields to assess the generality and robustness of the proposed formulation. 


\section{Stabilized mixed strain/displacement formulation for plasticity}

\subsection{Mixed $\varepsilon / \mathrm{u}$ formulation for plasticity}

The strong form of the continuous quasi-static solid mechanics problem can be stated as: given the prescribed body forces $\mathbf{f}$, find the displacement field $\mathbf{u}$ and the stress field $\boldsymbol{\sigma}$ such that:

$$
\begin{array}{rlll}
-\mathbf{C}^{-1}: \boldsymbol{\sigma}+\nabla^{s} \mathbf{u} & =\mathbf{0} & \text { in } \Omega \\
\nabla \cdot \boldsymbol{\sigma}+\mathbf{f} & =\mathbf{0} & \text { in } \Omega
\end{array}
$$

where $\Omega$ is the open and bounded domain of $\mathbb{R}^{\operatorname{dim}}$ occupied by the solid in a space of dim dimensions. The symbol $\boldsymbol{\nabla}^{s}(\cdot)$ is used to denote the symmetric gradient, whereas $\boldsymbol{\nabla} \cdot(\cdot)$ refers to the divergence operator. Eq. (1a) enforces both the geometric equation for linear kinematics and the non-linear constitutive relationship $\sigma=\mathbf{C}: \varepsilon$, with $\mathbf{C}=\mathbf{C}(\sigma)$ being the (secant) nonlinear constitutive tensor; Eq. (1b) is the balance of momentum Cauchy equation.

Equations (1a)-(1b) are subjected to appropriate Dirichlet and Neumann boundary conditions. In the following, we will assume these in the form of prescribed displacements $\mathbf{u}=0$ on $\partial \Omega_{u}$, and prescribed tractions $\overline{\mathbf{t}}$ on $\partial \Omega_{t}$, respectively, being $\partial \Omega_{u}$ and $\partial \Omega_{t}$ a partition of $\partial \Omega$.

This mixed formulation in terms of the stress and displacement fields, $\sigma / \mathbf{u}$, is classical and it has been used many times in the context of linear elasticity, where the constitutive tensor $\mathbf{C}=\mathbf{C}_{\mathbf{o}}$ is constant. However, this is not the most convenient format for the nonlinear problem. The reason

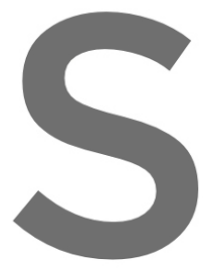
for this is that most of have been derived for t and they have a form

for linear kinematics.

Because of this, th
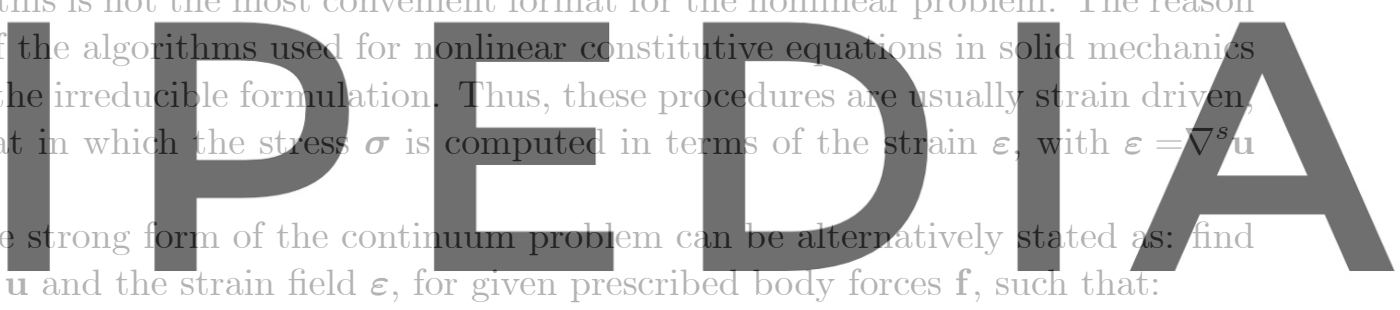

\section{Register for free at https//www.scipedia.coph: Eóndownloadithe version without the watermark

$$
\nabla \cdot[\mathrm{C}: \varepsilon]+\mathrm{f}=0 \quad \text { in } \Omega
$$

In small strain plasticity, the strain tensor $\varepsilon$ is decomposed additively as

$$
\varepsilon=\varepsilon_{e}+\varepsilon_{p}
$$

with $\varepsilon_{e}$ the elastic strain tensor and $\varepsilon_{p}$ the plastic strain tensor. The plasticity model is defined by appropriate evolution laws for the plastic strain. The constitutive equation is usually stated as

$$
\boldsymbol{\sigma}=\mathbf{C}_{o}: \varepsilon_{e}=\mathbf{C}_{o}:\left(\varepsilon-\varepsilon_{p}\right)
$$

The problem is closed once the expression of $\varepsilon_{p}$ is provided. In practice, an evolution law $\dot{\varepsilon}_{p}=\dot{\varepsilon}_{p}(\boldsymbol{\sigma})$ is formulated, the dot standing for the time derivative.

Using this constitutive equation, rather the secant one, the strong form of the plasticity problem may be written as

$$
\begin{aligned}
& -\mathbf{C}_{o}: \varepsilon+\mathbf{C}_{\mathbf{o}}: \nabla^{s} \mathbf{u}=\mathbf{0} \quad \text { in } \Omega \\
& \nabla \cdot\left[\mathbf{C}_{o}:\left(\varepsilon-\varepsilon_{p}\right)\right]+\mathbf{f}=\mathbf{0} \quad \text { in } \Omega
\end{aligned}
$$


Let $\mathcal{V}$ and $\mathcal{G}$ be the appropriate functional spaces where $\mathbf{u}$ and $\varepsilon$ are sought, respectively. Multiplying by appropriate test functions and integrating by parts the second equation, the associated weak form of the mixed problem can be stated as:

$$
\begin{aligned}
-\left(\boldsymbol{\gamma}, \mathbf{C}_{o}: \boldsymbol{\varepsilon}\right)+\left(\boldsymbol{\gamma}, \mathbf{C}_{o}: \nabla^{s} \mathbf{u}\right) & =0 & & \forall \boldsymbol{\gamma} \\
\left(\nabla^{s} \mathbf{v}, \mathbf{C}_{o}:\left(\varepsilon-\varepsilon_{p}\right)\right) & =(\mathbf{v}, \mathbf{f})+(\mathbf{v}, \overline{\mathbf{t}})_{\partial \Omega_{t}} & & \forall \mathbf{v}
\end{aligned}
$$

where $\mathbf{v} \in \mathcal{V}$ and $\gamma \in \mathcal{G}$ are the variations of the displacements and strain fields, respectively, with $\mathcal{V} \subset H^{1}(\Omega)^{\mathrm{dim}}$, this being the space of square integrable vector functions $\mathbf{v}$ which are at least square integrable and have square integrable first derivative, and $\mathcal{G} \subset L^{2}(\Omega)_{\mathrm{sym}}^{\operatorname{dim} \times \operatorname{dim}}$, this being the space of square integrable symmetric tensors $\gamma$. The inclusions $\mathcal{V} \subset H^{1}(\Omega)^{\operatorname{dim}}$ and $\mathcal{G} \subset L^{2}(\Omega)_{\mathrm{sym}}^{\operatorname{dim} \times \operatorname{dim}}$ are required because functions in $\mathcal{V}$ must vanish on $\partial \Omega_{u}$ and because more regularity might be needed for the evolution law $\dot{\varepsilon}_{p}=\dot{\varepsilon}_{p}(\sigma)$ to make sense. Parenthesis $(\cdot, \cdot)$ denotes the inner product in $L^{2}(\Omega)$, and $(\mathbf{v}, \bar{t}) \partial \Omega_{t}$ denotes the integral of the product of $\mathbf{v}$ and $\overline{\mathbf{t}}$ over $\partial \Omega_{t}$.

Let us consider a finite element partition of the domain $\Omega$ from which we can construct finite element spaces $\mathcal{V}_{h} \subset \mathcal{V}$ and $\mathcal{G}_{h} \subset \mathcal{G}$ in the usual manner. A generic element size of this partition will be denoted by $h$, and this subscript will be used to refer to finite element functions. The discrete Galerkin finite element counterpart problem is defined as:
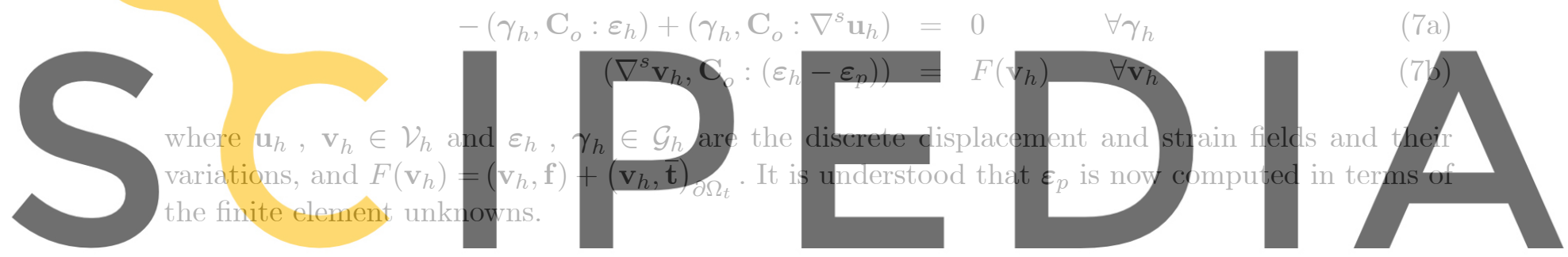

Remark The strong form (5a)-(5b), as well as the corresponding discrete weak form (7a)-(7b), are

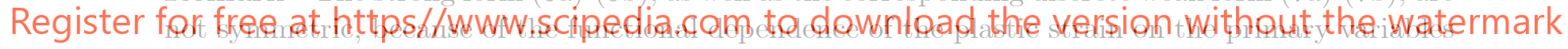

of the problem. This can be remedied in two ways.

$$
\begin{aligned}
& \text { The constitutive equation (4) can be equivalently expressed, for example, as } \\
& \qquad \boldsymbol{\sigma}=\left[\mathbf{C}_{o}-\frac{\left(\mathbf{C}_{o}: \varepsilon_{p}\right) \otimes\left(\mathbf{C}_{o}: \boldsymbol{\varepsilon}_{p}\right)}{\boldsymbol{\varepsilon}: \mathbf{C}_{o}: \boldsymbol{\varepsilon}_{p}}\right]: \boldsymbol{\varepsilon}=\mathbf{C}: \boldsymbol{\varepsilon}
\end{aligned}
$$

where the (secant) nonlinear constitutive tensor $\mathbf{C}$ is symmetric by construction. The form (8) can be fitted directly in the strong form $(2 \mathrm{a})-(2 \mathrm{~b})$ to provide the corresponding symmetric discrete weak form:

$$
\begin{array}{rlrl}
-\left(\gamma_{h}, \mathbf{C}: \varepsilon_{h}\right)+\left(\boldsymbol{\gamma}_{h}, \mathbf{C}: \nabla^{s} \mathbf{u}_{h}\right) & =0 & & \forall \boldsymbol{\gamma}_{h} \\
\left(\nabla^{s} \mathbf{v}_{h}, \mathbf{C}: \varepsilon_{h}\right) & =F\left(\mathbf{v}_{h}\right) & \forall \mathbf{v}_{h}
\end{array}
$$

This form is identical to the discrete form obtained in references [1] and [2].

The second alternative is as follows. The constitutive equation (4) may be expressed in rate form as

$$
\dot{\boldsymbol{\sigma}}=\mathbf{C}_{e p}: \dot{\varepsilon}
$$


where $\mathbf{C}_{e p}$ is the (tangent) elasto-plastic constitutive tensor, symmetric for associative plasticity (see Subsection 4.3).

Making use of this, and stating all the governing equations in rate form, the strong form of the problem can be written as

$$
\begin{aligned}
-\mathbf{C}_{e p}: \dot{\varepsilon}+\mathbf{C}_{e p}: \nabla^{s} \dot{\mathbf{u}} & =\mathbf{0} & & \text { in } \Omega \\
\nabla \cdot\left[\mathbf{C}_{e p}: \dot{\boldsymbol{\varepsilon}}\right]+\dot{\mathbf{f}} & =\mathbf{0} & & \text { in } \Omega
\end{aligned}
$$

This form is symmetric and leads to the symmetric discrete weak form:

$$
\begin{array}{rlrl}
-\left(\gamma_{h}, \mathbf{C}_{e p}: \dot{\varepsilon}_{h}\right)+\left(\gamma_{h}, \mathbf{C}_{e p}: \nabla^{s} \dot{\mathbf{u}}_{h}\right) & =0 & & \forall \gamma_{h} \\
\left(\nabla^{s} \mathbf{v}_{h}, \mathbf{C}_{e p}: \dot{\varepsilon}_{h}\right) & =\dot{F}\left(\mathbf{v}_{h}\right) & \forall \mathbf{v}_{h}
\end{array}
$$

where $\dot{F}\left(\mathbf{v}_{h}\right)$ is the counterpart of $F\left(\mathbf{v}_{h}\right)$ when loads are expressed in rate form.

\subsection{Variational Multiscale Stabilization}

The inf-sup condition [10] establishes that the stability of the discrete formulation depends on the appropriate choice of the finite element spaces $\mathcal{V}_{h}$ and $\mathcal{G}_{h}$. Even for linear elasticity, standard Galerkin mixed elements with continuous equal order P1P1 (linear/linear) and Q1Q1 (bilinear/bilinear) interpolation for both fields do not satisfy the condition and, therefore, are not

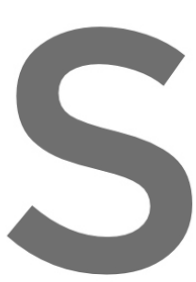
stable. For the $\varepsilon /$ u

the displacement field condition is to modify that can provide the nece problem nor degradin i Variational Multiscale Stabilization (VMS).

VMS was developed in first instance by [11] and then generalized in [12]. This technique

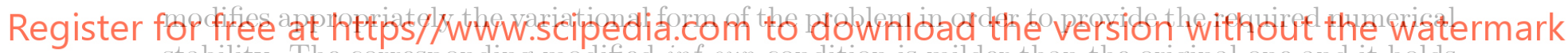
stability. The corresponding modified inf-sup condition is milder than the original one and it holds for most common equal order finite element spaces [13].

The multiscale procedure decomposes the solution $(\varepsilon, \mathbf{u})$ into a resolvable finite element scale $\left(\varepsilon_{h}, \mathbf{u}_{h}\right)$ and an unresolvable subscale $(\tilde{\varepsilon}, \tilde{\mathbf{u}})$, so that:

$$
\begin{aligned}
\varepsilon & =\varepsilon_{h}+\tilde{\varepsilon} \\
\mathbf{u} & =\mathbf{u}_{h}+\tilde{\mathbf{u}}
\end{aligned}
$$

This extends the solution spaces for the displacements and the strains to $\mathcal{V} \simeq \mathcal{V}_{h} \oplus \widetilde{\mathcal{V}}$ and $\mathcal{G} \simeq \mathcal{G}_{h} \oplus \widetilde{\mathcal{G}}$ where $\widetilde{\mathcal{V}}$ and $\widetilde{\mathcal{G}}$ are the functional spaces for the subscale variables $(\tilde{\boldsymbol{\varepsilon}}, \tilde{\mathbf{u}})$ and their test functions $(\tilde{\gamma}, \tilde{\mathbf{v}})$. It can also be assumed that $\tilde{\varepsilon}$ and $\tilde{\gamma}$ vanish on the boundary $\partial \Omega$.

The plastic strain $\varepsilon_{p}$ is non-linearly dependent on the stress field, this in turn being dependent on the strain field through the constitutive equation. Since the strain field $\varepsilon$ includes a subscale contribution, then also the plastic strain tensor $\varepsilon_{p}$ could present a corresponding subscale part. However, since the subscale contribution is assumed to be small with respect the resolvable scale, the plastic strain will be approximated as:

$$
\varepsilon_{p}=\varepsilon_{p}(\boldsymbol{\sigma}) \approx \varepsilon_{p}\left(\boldsymbol{\sigma}_{h}\right)
$$


This implies that the constitutive model is evaluated only with the resolvable part of the strain:

$$
\boldsymbol{\sigma}_{h}=\mathbf{C}_{o}:\left[\varepsilon_{h}-\varepsilon_{p}\left(\boldsymbol{\sigma}_{h}\right)\right]
$$

It would be possible however to account for the effect of the subscales in this expression, at the expense of increasing the non-linearity of the problem.

Considering the scale splitting, the discrete problem corresponding to Eqs. (6a)-(6b) is now:

$$
\begin{aligned}
-\left(\boldsymbol{\gamma}_{h}, \mathbf{C}_{o}:\left[\varepsilon_{h}+\widetilde{\varepsilon}\right]\right)+\left(\gamma_{h}, \mathbf{C}_{o}: \nabla^{s}\left(\mathbf{u}_{h}+\widetilde{\mathbf{u}}\right)\right) & =0 & & \forall \gamma_{h} \\
\left(\nabla^{s} \mathbf{v}_{h}, \mathbf{C}_{o}:\left[\varepsilon_{h}+\widetilde{\varepsilon}-\varepsilon_{p}\right]\right) & =F\left(\mathbf{v}_{h}\right) & & \forall \mathbf{v}_{h} \\
-\left(\widetilde{\boldsymbol{\gamma}}, \mathbf{C}_{o}:\left[\varepsilon_{h}+\widetilde{\varepsilon}\right]\right)+\left(\widetilde{\gamma}, \mathbf{C}_{o}: \nabla^{s}\left(\mathbf{u}_{h}+\widetilde{\mathbf{u}}\right)\right) & =0 & & \forall \widetilde{\gamma} \\
-\left(\nabla^{s} \widetilde{\mathbf{v}}, \boldsymbol{\nabla} \cdot \mathbf{C}_{o}:\left[\varepsilon_{h}+\widetilde{\boldsymbol{\varepsilon}}-\varepsilon_{p}\right]\right)+(\widetilde{\mathbf{v}}, \mathbf{f}) & =0 & & \forall \widetilde{\mathbf{v}}
\end{aligned}
$$

where due to linear independence, each of the equations in (6a) and (6b) unfolds into two equations, one related to each scale considered.

Rewriting the third and fourth equations, tested against the subscale test functions, and assuming that the subscale $(\tilde{\varepsilon}, \tilde{\mathbf{u}})$ vanishes on the boundary, it follows that

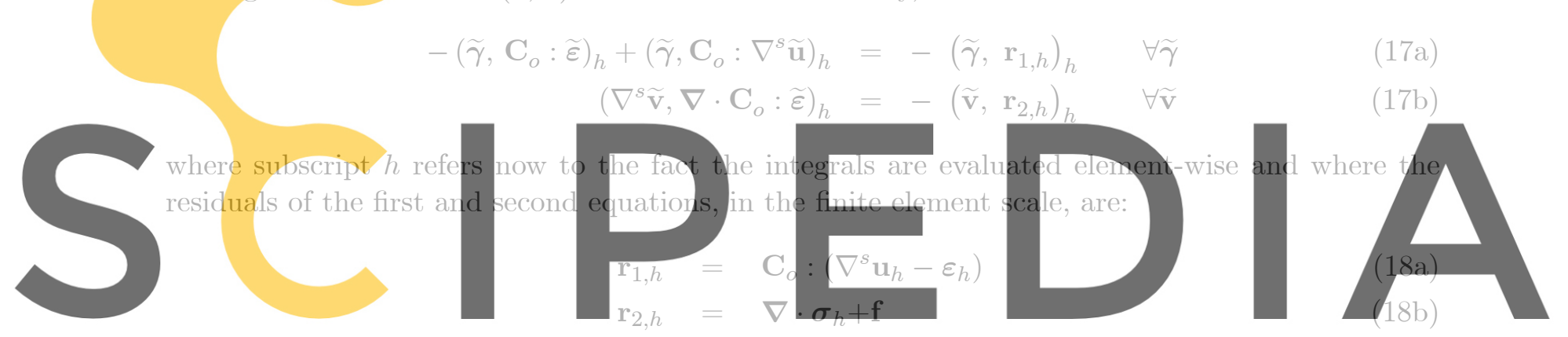

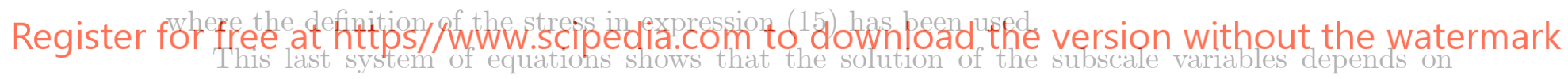

the residuals upon substitution of the resolvable FE solution in the strong form of the problem.

Therefore, following the work of [14], the residual based subscales strain can be localized within each finite element and expressed as

$$
\begin{aligned}
\widetilde{\boldsymbol{\varepsilon}} & =\tau_{\varepsilon} \mathbf{C}_{o}^{-1}: P\left(\mathbf{r}_{1, h}\right)=\tau_{\varepsilon} P\left(\nabla^{s} \mathbf{u}_{h}-\boldsymbol{\varepsilon}_{h}\right) \\
\tilde{\mathbf{u}} & =\tau_{u} P\left(\mathbf{r}_{2, h}\right)=\tau_{u} P\left(\boldsymbol{\nabla} \cdot \boldsymbol{\sigma}_{h}+\mathbf{f}\right)
\end{aligned}
$$

where $P(\cdot)$ represents an appropriate projection operator onto the space of subscales and $\tau_{\varepsilon}, \tau_{u}$ are computed as

$$
\tau_{\varepsilon}=c_{\varepsilon} \frac{h}{L} \frac{\mu}{\mu_{o}} \quad \text { and } \quad \tau_{u}=c_{u} \frac{h L}{\mu}
$$

where $c_{\varepsilon}$ and $c_{u}$ are positive constants, $\mu$ is a mechanical parameter of the problem, usually chosen as the ratio between the norms of the deviatoric stress and total strain tensors, $\mu=\|\operatorname{dev} \boldsymbol{\sigma}\| /\|\operatorname{dev} \varepsilon\|$, $\mu_{o}$ being its initial elastic value. For nonlinear constitutive models, this ratio is non-constant and it varies along the deformation process. Dimension $h$ is the size of the finite element and $L$ is a characteristic length of the problem. The expression given by (20) has been chosen according 
to the optimal convergence results obtained for equal interpolation in [14], since in the following we precisely assume equal continuous interpolation for displacements and strains. For the sake of clarity, $h$ will be assumed constant for all elements, even if in practice expressions (20) are evaluated element-wise.

To complete the stabilization method, an appropriate projection operator has to be selected in order to be able to compute the subscale variables.

\subsubsection{ASGS}

In the Algebraic Subgrid Scale Stabilization [12], the projection operator is taken as the identity when applied to finite element residuals, that is, $P(\mathbf{r})=\mathbf{r}$, and, therefore, the subscales read:

$$
\begin{aligned}
\widetilde{\boldsymbol{\varepsilon}} & =\tau_{\varepsilon}\left(\nabla^{s} \mathbf{u}_{h}-\boldsymbol{\varepsilon}_{h}\right) \\
\tilde{\mathrm{u}} & =\tau_{u}\left(\nabla \cdot \sigma_{h}+\mathrm{f}\right)
\end{aligned}
$$

Introducing these strain and displacement subscales in Eqs. (16a) and (16c), integrating by parts the last term in the first equation and recalling that the subscales vanish on the boundary, the mixed discrete system of equations can be written as
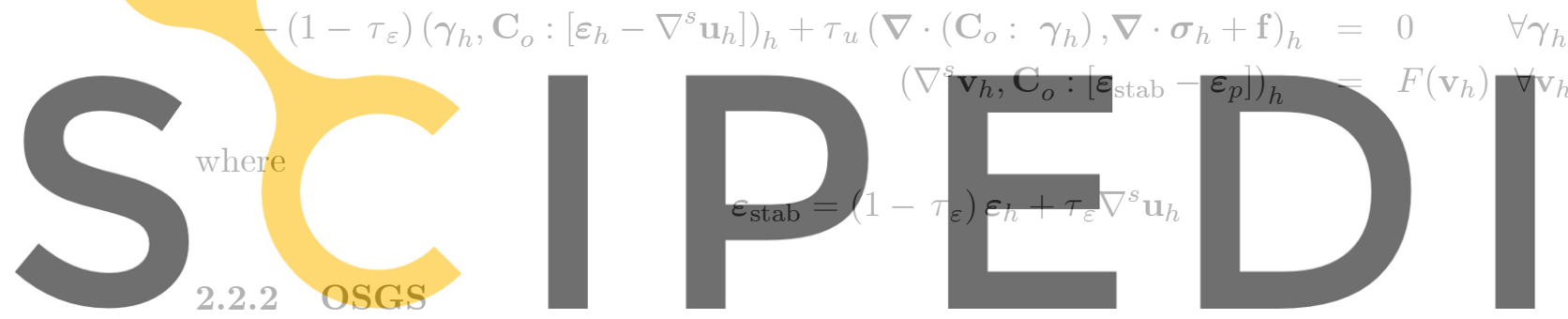

(22a)

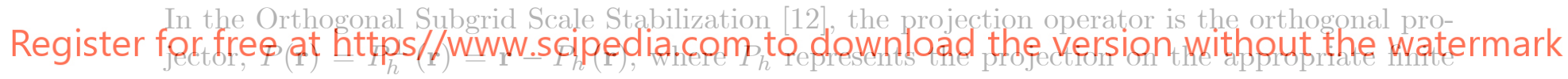

element space. It is performed taking advantage of the orthogonality condition

$$
\left(\boldsymbol{\eta}_{h}, \boldsymbol{\Pi}_{\mathbf{r}}-\mathbf{r}\right)=0 \quad \forall \boldsymbol{\eta}_{h}
$$

where $\Pi_{\mathbf{r}}$ is the projected value of $\mathbf{r}$ on the finite element space and $\boldsymbol{\eta}_{h}$ belongs either to $\mathcal{V}_{h}$ or $\mathcal{G}_{h}$.

According to this, the subscale variables $\tilde{\mathbf{u}}$ and $\tilde{\varepsilon}$ are approximated as:

$$
\begin{aligned}
\widetilde{\varepsilon} & =\tau_{\varepsilon}\left(\nabla^{s} \mathbf{u}_{h}-P_{h}\left(\nabla^{s} \mathbf{u}_{h}\right)\right) \\
\tilde{\mathbf{u}} & =\tau_{u}\left(\boldsymbol{\nabla} \cdot \boldsymbol{\sigma}_{h}-P_{h}\left(\boldsymbol{\nabla} \cdot \boldsymbol{\sigma}_{h}\right)\right)
\end{aligned}
$$

where it has been assumed that $P_{h}(\mathbf{f})=\mathbf{f}$.

Back-substituting in the set of equations of the problem, the problem to be solved is

$$
\begin{aligned}
-\left(\gamma_{h}, \mathbf{C}_{o}: \varepsilon_{h}\right)-\tau_{\varepsilon}\left(\gamma_{h}, \mathbf{C}_{o}:\left[\nabla^{s} \mathbf{u}_{h}-P_{h}\left(\nabla^{s} \mathbf{u}_{h}\right)\right]\right)_{h}+ & \\
\left(\gamma_{h}, \mathbf{C}_{o}: \nabla^{s} \mathbf{u}_{h}\right)-\tau_{u}\left(\nabla \cdot \mathbf{C}_{o}: \gamma_{h}, \nabla \cdot \boldsymbol{\sigma}_{h}-P_{h}\left(\nabla \cdot \boldsymbol{\sigma}_{h}\right)\right)_{h} & =0 \\
\left(\nabla^{s} \mathbf{v}_{h}, \mathbf{C}_{o}:\left[\varepsilon_{h}-\boldsymbol{\varepsilon}_{p}\right]\right)+\tau_{\varepsilon}\left(\nabla^{s} \mathbf{v}_{h}, \mathbf{C}_{o}:\left[\nabla^{s} \mathbf{u}_{h}-P_{h}\left(\nabla^{s} \mathbf{u}_{h}\right)\right]\right)_{h} & =F\left(\mathbf{v}_{h}\right)
\end{aligned}
$$




\subsubsection{Modified OSGS}

The formulation given by (26a)-(26b) has a numerical performance very similar to the ASGS method given by (21a)-(21b). However, when localization occurs the formulation that has been found most robust, and that has been used in the numerical examples, is the modification of (26a)-(26b) described next.

First, it may be assumed that

$$
P_{h}\left(\nabla^{s} \mathbf{u}_{h}\right) \approx \varepsilon_{h}
$$

which essentially means that the strain subscale is assumed to be given by the ASGS formulation rather than by the OSGS one. This avoids the need to compute the projection $P_{h}\left(\nabla^{s} \mathbf{u}_{h}\right)$, but it is not crucial at all, neither for stability nor for accuracy.

The second modification is the important one. If $\operatorname{dev}(\mathbf{a})$ and $\frac{1}{3} \operatorname{tr}(\mathbf{a}) \mathbf{1}$, with $\mathbf{1}$ the second order identity tensor, are respectively the deviatoric and volumetric components of a tensor a, and $P_{h}^{\frac{1}{h}}=$ $I-P_{h}$ is the projection orthogonal to the appropriate finite element space, the last term in (26a) may be written as
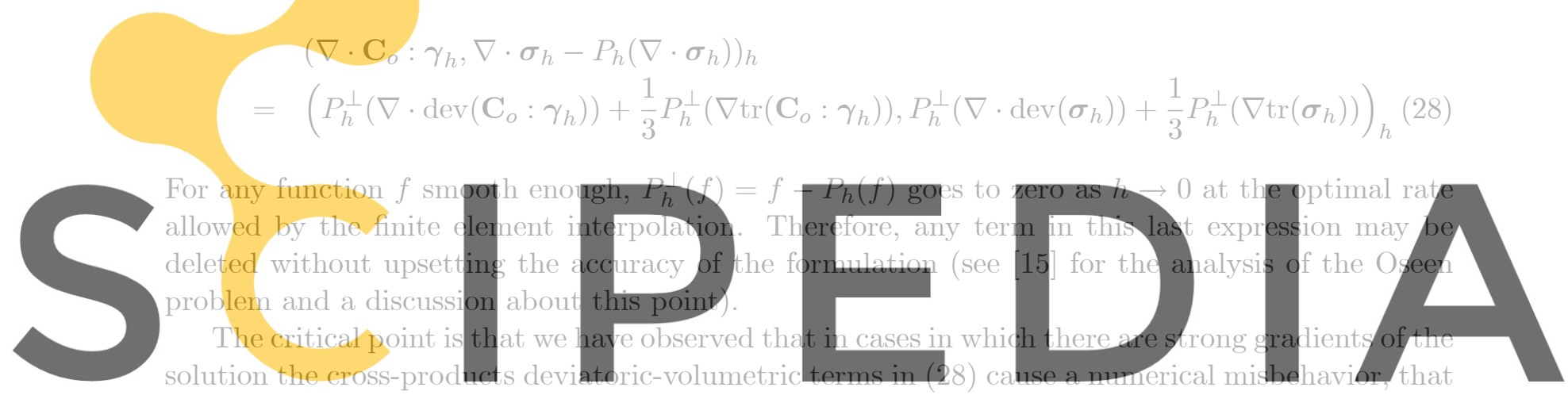

manifests in the plasticity problem as a (small) volumetric locking. A similar situation was found

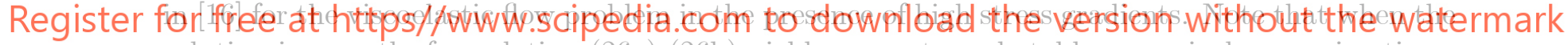
solution is smooth, formulation (26a)-(26b) yields accurate and stable numerical approximations. Moreover, the deviatoric-deviatoric product in (28) leads to a positive-definite term and in principle should enhance stability, but we have found no instability problems when it is omitted.

In view of these observations, only the volumetric-volumetric term is kept in (28), i.e.,

$$
\left(\nabla \cdot \mathbf{C}_{o}: \gamma_{h}, \nabla \cdot \boldsymbol{\sigma}_{h}-P_{h}\left(\nabla \cdot \boldsymbol{\sigma}_{h}\right)\right)_{h} \approx \frac{1}{9}\left(\nabla \operatorname{tr}\left(\mathbf{C}_{o}: \gamma_{h}\right), \nabla \operatorname{tr}\left(\boldsymbol{\sigma}_{h}\right)-P_{h}\left(\nabla \operatorname{tr}\left(\boldsymbol{\sigma}_{h}\right)\right)\right)_{h}
$$

This, together with (27), leads to the modified OSGS formulation:

$$
\begin{aligned}
-\left(1-\tau_{\varepsilon}\right)\left(\gamma_{h}, \mathbf{C}_{o}:\left[\varepsilon_{h}-\nabla^{s} \mathbf{u}_{h}\right]\right)+\tau_{u} \frac{1}{9}\left(\nabla \operatorname{tr}\left(\mathbf{C}_{o}: \boldsymbol{\gamma}_{h}\right), \nabla \operatorname{tr}\left(\boldsymbol{\sigma}_{h}\right)-P_{h}\left(\nabla \operatorname{tr}\left(\boldsymbol{\sigma}_{h}\right)\right)\right) & =0 \quad \forall \boldsymbol{\gamma}_{h}(30 \mathrm{a}) \\
\left(\nabla^{s} \mathbf{v}_{h}, \mathbf{C}_{o}:\left[\varepsilon_{\text {stab }}-\boldsymbol{\varepsilon}_{p}\right]\right)_{h} & =F\left(\mathbf{v}_{h}\right) \forall \mathbf{v}_{h}(30 \mathrm{~b})
\end{aligned}
$$

with $\varepsilon_{\text {stab }}$ given by (23). As mentioned above, this is the numerical formulation used in the numerical examples. 


\section{Implementation and computational aspects}

In the presented mixed formulation, the presence of the non-linear plastic strains requires an iterative procedure to deal with the nonlinearity of the problem. Iterative solution schemes, such as Picard or Newton-Raphson methods, need to be introduced. Constitutive laws involving plasticity are usually written in terms of rate equations and, consequently, the matrices involved in the resulting algebraic set of equations are tangent to the strain-stress path. Hence, the use of the Newton-Raphson scheme will be considered in the following. The advantage of such method is a quadratic convergence rate in the iteration at each time step. Its use requires the computation of the Jacobian matrix of the system of equation at each iteration of every time step.

\subsection{ASGS}

In the case of the ASGS scheme, differentiating the system of equations (22a)-(22b) at iteration $i$ of time step $n+1$, the Jacobian matrix presents the structure:

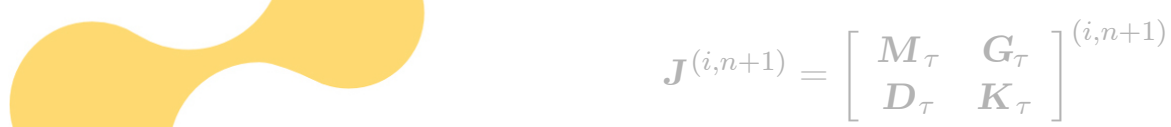

where $M$ is a projection mass-like matrix, $G$ is a gradient matrix, $D$ is a divergence matrix and $\mathbb{K}$ is the stiffness matrix. The subscript $\tau$ refers to the fact that those matrices incorporates stabilization terms. Differentiating plastic strain depends
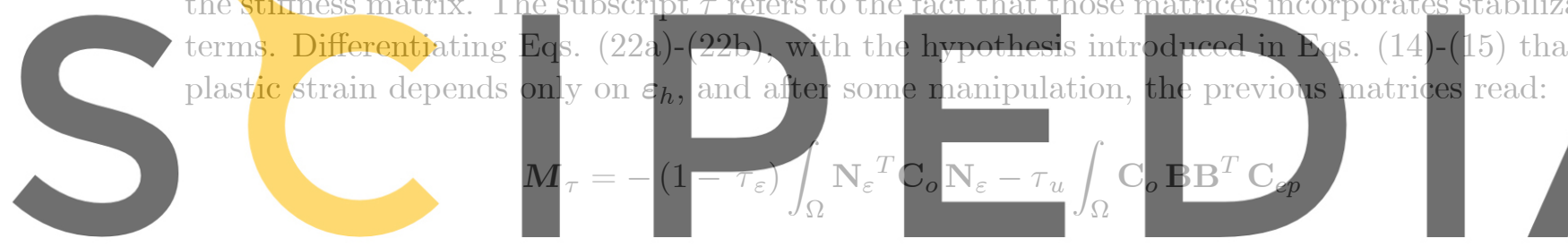

$G_{\tau}=\left(1-\tau_{\varepsilon}\right)$
Register for free at https//www.scipedia.com to download the

$$
\begin{gathered}
D_{\tau}=\int_{\Omega} B^{T}\left[\mathbf{C}_{e p}-\tau_{\varepsilon} \mathbf{C}_{o}\right] \mathbb{N}_{u} \\
\boldsymbol{K}_{\tau}=\tau_{\varepsilon} \int_{\Omega} \mathbf{B}^{T} \mathbf{C}_{o} \mathbf{B}
\end{gathered}
$$

where $\mathbf{N}_{\varepsilon}$ and $\mathbf{N}_{u}$ are the matrices of shape functions of the respective strain and displacement fields and $\mathbf{B}$ is the matrix of the gradient of those shape functions. The resulting algebraic system of equations is, in general, not symmetric. Note that disregarding the terms due to plasticity, the system matrix is symmetric and it coincides with the one presented in [1], [2]. In the general elasto-plastic case, matrix $\mathbf{C}_{e p}$ is tangent to the stress-strain path. In Subsection 4.3 we introduce it and describe how to compute it so that it is tangent to the time-discrete stress-strain path.

\subsection{Modified OSGS}

The modified OSGS implementation is identical to the ASGS implementation, except for the additional projection of the gradient of the trace of the nodal stresses and the second term in (32), which in this case only contains the volumetric components of $\mathbf{C}_{o}$ and $\mathbf{B}$. 
Concerning the additional equation due to the projection, it has the structure

$$
M_{\Pi} \Pi+D_{\Pi} \Sigma=0
$$

where $\boldsymbol{\Pi}$ are the nodal values of the projected variable (gradient of the trace of the stress) and $\boldsymbol{\Sigma}$ of the stresses, and where

$$
\boldsymbol{M}_{\boldsymbol{\Pi}}=-\int_{\Omega} \mathbf{N}_{u}^{T} \mathbf{N}_{u}
$$

The Jacobian in (31) has to be completed with the last row corresponding to (36) and the columns accounting for the effect of $\boldsymbol{\Pi}$ in the first equation. Alternatively to this procedure, a staggered scheme can be devised. First, the projection of the stresses $\boldsymbol{\Pi}^{(0, n+1)}$ is computed at the beginning of the time step. Then, the approximation $\boldsymbol{\Pi}^{(i, n+1)} \approx \boldsymbol{\Pi}^{(0, n+1)}$ is used for the solution of $\left(\varepsilon_{h}, \mathbf{u}_{h}\right)$. This scheme is preferred with respect to the monolithic one due to the reduced computation time required, almost identical to that of the ASGS scheme.

\section{Pressure dependent plasticity. The Drucker-Prager model}

\subsection{Yield criterion}

The Drucker-Prager plasticity model may be constructed as a linear combination of a pure isochoric plasticity model and a pure pressure plasticity model, in the form:

$$
f(\boldsymbol{\sigma}, q)=\left[\sqrt{\frac{3}{2}}\|\operatorname{dev} \boldsymbol{\sigma}\|-r^{d}(q)\right]+a \tan \phi\left[\frac{1}{3} \operatorname{tr} \boldsymbol{\sigma}-r^{p}(q)\right]=0
$$

where the angle of friction $\phi$ is introduced to relate the admissible deviatoric stresses to the pressure. Here, $r^{d}=r^{d}(q)$ and $r^{p}=r^{p}(q)$ are the admissible stresses of the deviatoric and volumetric parts of the model, respectively, and $q$ is a stress-like internal variable that controls the hardening/softening of the model. In this work, the pressure threshold is taken as $r^{p}=0$ to allow a direct comparison between J2 incompressible plasticity and Drucker-Prager plasticity.

In the principal stress Haigh-Westergaard space, the Drucker-Prager yield surface appears as a symmetric cone with the axis coinciding with the hydrostatic pressure and a circular trace on the octahedral plane (see Figure 1). The parameter $a= \pm 1$ controls the sign of the pressure part and the orientation of the cone. For $a=1$, the cone is open in the triaxial compression end, while for $a=-1$, it is open for triaxial tension.

The deviatoric stress threshold is expressed as:

$$
r^{d}(q)=\sigma_{y}-q(\xi)
$$

where $\xi$ is an internal strain-like parameter and $q(\xi)$ is the hardening/softening function:

$$
q(\xi)=\left\{\begin{array}{lll}
H \xi & \text { for } & 0 \leq \xi \leq \frac{\sigma_{y}}{H} \\
0 & \text { for } & \frac{\sigma_{y}}{H} \leq \xi \leq \infty
\end{array}\right.
$$

for linear softening, whereas, in the case of exponential softening, it takes the form:

$$
q(\xi)=\sigma_{y}\left(1-\exp \left(\frac{-2 H}{\sigma_{y}} \xi\right)\right) \quad \text { for } \quad 0 \leq \xi \leq \infty
$$




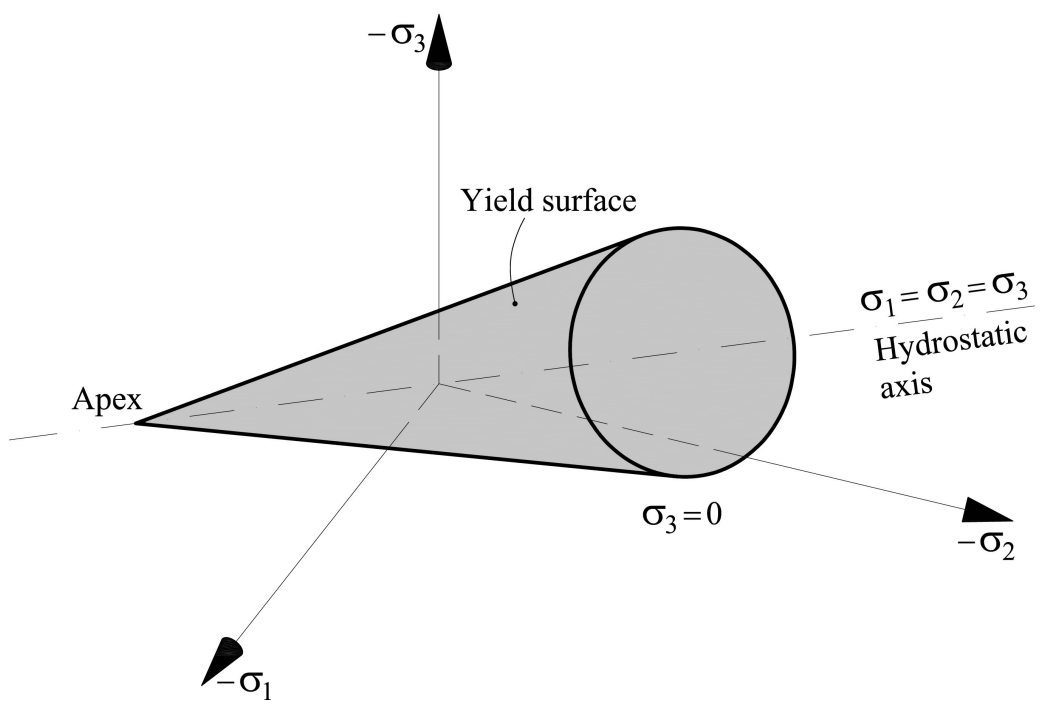

Figure 1: Yield surface for Drucker-Prager plasticity model in the stress space $(a=1)$

where $\sigma_{y}$ is the initial deviatoric threshold and $H$ is the softening parameter.

Making $\rho=1 /(1+\tan \phi)$, the yield surface may be rewritten as:

$$
f(\boldsymbol{\sigma}, q)=\rho\left(\sqrt{\frac{3}{2}}\|\operatorname{dev} \boldsymbol{\sigma}\|-\left(\sigma_{y}-q(\xi)\right)\right)+a(1-\rho) \frac{1}{3} \operatorname{tr} \boldsymbol{\sigma}=0
$$

In the following, linear isotropic elasticity is assumed, with the elastic constitutive tensor given by:

$$
\mathbf{C}_{o}=K \mathbf{1} \otimes \mathbf{1}+2 G\left(\mathbf{I}-\frac{1}{3} \mathbf{1} \otimes \mathbf{1}\right)
$$

where $K$ is the bulk modulus, $G$ is the shear modulus and $\mathbf{1}$ and $\mathbf{I}$ are the second and fourth order identity tensors, respectively.

\subsection{Return mapping algorithm}

Assuming associative plasticity and the existence of a plastic potential that coincides with the definition of the admissible stress surface $f(\boldsymbol{\sigma}, q)$, the evolution equations for the plastic variables read:

$$
\begin{aligned}
& \dot{\varepsilon}_{p}=\dot{\gamma} \partial_{\boldsymbol{\sigma}} f(\boldsymbol{\sigma}, q) \\
& \dot{\xi}=\dot{\gamma} \partial_{q} f(\boldsymbol{\sigma}, q)
\end{aligned}
$$

where $\dot{\gamma}$ is the plastic multiplier or plastic consistency parameter.

Additionally, given the Karush-Kuhn-Tucker and consistency conditions:

$$
\begin{gathered}
\gamma \geq 0, \quad f(\boldsymbol{\sigma}, q) \leq 0, \quad \gamma f(\boldsymbol{\sigma}, q)=0 \\
\text { if } \quad f(\boldsymbol{\sigma}, q)=0 \quad \dot{\gamma} \geq 0, \quad \dot{f}(\boldsymbol{\sigma}, q) \leq 0 \quad \text { and } \quad \dot{\gamma} \dot{f}(\boldsymbol{\sigma}, q)=0
\end{gathered}
$$


Substituting the definition of the failure surface and differentiating, the plastic multiplier $\dot{\gamma}$ is computed as [17]:

$$
\dot{\gamma}=\frac{1}{D} \partial_{\sigma} f: \mathbf{C}_{o}: \dot{\varepsilon}
$$

with

$$
D=\left\langle\partial_{\sigma} f: \mathbf{C}_{o}: \partial_{\sigma} f+\partial_{q} f \frac{d q}{d \xi} \partial_{q} f\right\rangle
$$

The time derivative of the evolution equations of the plastic variables can be approximated introducing a Backward-Euler scheme with time steps of length $\Delta t$. Let us consider the time span $\left[t_{n}, t_{n+1}\right]$, with $t_{n+1}=t_{n}+\Delta t$, where variables are known at step $(n)$ and must be computed at step $(n+1)$. Then, the discrete-in-time version of (43) reads:

$$
\begin{aligned}
& \dot{\varepsilon}_{p} \approx \frac{\varepsilon_{p}^{(n+1)}-\varepsilon_{p}^{(n)}}{\Delta t}=\frac{\gamma^{(n+1)}-\gamma^{(n)}}{\Delta t}\left[\rho \sqrt{\frac{3}{2}} \frac{\operatorname{dev} \boldsymbol{\sigma}^{(n+1)}}{\left\|\operatorname{dev} \boldsymbol{\sigma}^{(\mathrm{n}+1)}\right\|}+\frac{a(1-\rho)}{3} \mathbf{1}\right] \\
& \dot{\xi} \approx \frac{\xi^{(n+1)}-\xi^{(n)}}{\Delta t}=\rho \frac{\gamma^{(n+1)}-\gamma^{(n)}}{\Delta t}
\end{aligned}
$$

The trial state is defined at step $n+1$ with the plasticity variables $\varepsilon_{p}$ and $\xi$ frozen at step $n$. Therefore, the trial stresses are:

$$
\begin{aligned}
\boldsymbol{\sigma}_{\text {trial }}^{(n+1)} & =\mathbf{C}_{o}:\left(\varepsilon^{(n+1)}-\varepsilon_{p}^{(n)}\right) \\
q_{\text {trial }}^{(n+1)} & =q^{(n)}
\end{aligned}
$$

The trial yielding function is:

$$
f_{\text {trial }}^{(n+1)}=\rho\left(\sqrt{\frac{3}{2}}\left\|\operatorname{dev} \boldsymbol{\sigma}_{\text {trial }}^{(n+1)}\right\|-\left(\sigma_{y}-q_{\text {trial }}^{(n+1)}\right)\right)+a(1-\rho)\left(\frac{1}{3} \operatorname{tr} \boldsymbol{\sigma}_{\text {trial }}^{(n+1)}\right)
$$

Plasticity occurs if $f_{\text {trial }}^{(n+1)} \geq 0$. The update of the stress is then

$$
\boldsymbol{\sigma}^{(n+1)}=\boldsymbol{\sigma}_{\text {trial }}^{(n+1)}-\Delta \gamma^{(n+1)} \mathbf{C}_{o}: \partial_{\boldsymbol{\sigma}} f
$$

which can be particularized for the Drucker-Prager criterion as:

$$
\boldsymbol{\sigma}^{(n+1)}=\boldsymbol{\sigma}_{\text {trial }}^{(n+1)}-\Delta \gamma^{(n+1)}\left[a(1-\rho) K \mathbf{1}+2 G \rho \sqrt{\frac{3}{2}} \frac{\operatorname{dev} \boldsymbol{\sigma}_{\text {trial }}^{(n+1)}}{\left\|\operatorname{dev} \boldsymbol{\sigma}_{\text {trial }}^{(n+1)}\right\|}\right]
$$

The change of plastic multiplier $\Delta \gamma^{(n+1)}=\gamma^{(n+1)}-\gamma^{(n)}$ is computed with the discrete counterpart of (46) as:

$$
\Delta \gamma^{(n+1)}=\frac{f_{\text {trial }}^{(n+1)}}{(1-\rho)^{2} K+3 G \rho^{2}+\left.\rho^{2} \frac{d q}{d \xi}\right|^{(n+1)}}
$$




\subsection{Constitutive Elasto-Plastic Tangent operator}

On one hand, the constitutive elasto-plastic tensor in continuous form is [17]:

$$
\mathbf{C}_{e p}=\mathbf{C}_{o}-\mathbf{C}_{p}=\mathbf{C}_{o}-\frac{1}{D}\left(\mathbf{C}_{o}: \partial_{\boldsymbol{\sigma}} f\right) \otimes\left(\mathbf{C}_{o}: \partial_{\boldsymbol{\sigma}} f\right)
$$

On the other hand, considering the discrete Backward Euler time integration, the algorithmic consistent constitutive elasto-plastic tensor [18] can be computed as:

$$
\mathbf{C}_{e p}^{(n+1)}=\frac{\Delta \boldsymbol{\sigma}^{(n+1)}}{\Delta \varepsilon^{(n+1)}}
$$

Carrying out the differentiation, it yields:

$$
\begin{aligned}
\mathbf{C}_{e p}^{(n+1)}= & \mathbf{C}_{o} \\
& -\frac{1}{D^{(n+1)}}\left[\rho 2 G \sqrt{\frac{3}{2}} \mathbf{n}_{d, \text { trial }}^{(n+1)}+a(1-\rho) K \mathbf{1}\right] \otimes\left[\rho 2 G \sqrt{\frac{3}{2}} \mathbf{n}_{d, \text { trial }}^{(n+1)}+a(1-\rho) K \mathbf{1}\right] \\
& -\Delta \gamma^{(n+1)}(2 G)^{2} \rho \sqrt{\frac{3}{2}} \frac{1}{\left\|\operatorname{dev} \boldsymbol{\sigma}_{\text {trial }}^{(n+1)}\right\|}\left[\left(\mathbf{I}-\frac{1}{3} \mathbf{1} \otimes \mathbf{1}\right)-\mathbf{n}_{d, \text { trial }}^{(n+1)} \otimes \mathbf{n}_{d, \text { trial }}^{(n+1)}\right]
\end{aligned}
$$

where $D^{(n+1)}$ is the discrete counterpart of $(47)$ :

$$
D^{(n+1)}=\left[(1-\rho)^{2} K+\rho^{2} 3 G\right]-\rho^{3} \frac{d q\left(\xi^{(n)}+\rho \Delta \gamma^{(n+1)}\right)}{d \xi}
$$

and $\mathbf{n}_{d, \text { trial }}^{(n+1)}$ is the unit vector in the trial deviatoric stress direction:

$$
\mathbf{n}_{d, \text { trial }}^{(n+1)}=\frac{\operatorname{dev} \boldsymbol{\sigma}_{\text {trial }}^{(n+1)}}{\left\|\operatorname{dev} \boldsymbol{\sigma}_{\text {trial }}^{(n+1)}\right\|}
$$

\subsection{Apex return mapping}

The apex of the Drucker-Prager cone is a singular point in the yield surface. This means that the cases when the return mapping is to the apex, rather than to the regular lateral surface of the cone, have to be identified and an "ad-hoc" procedure is necessary [19, 20, 21]. In the standard return mapping algorithm, Eq. (52), considering the deviatoric part and taking norms, it is:

$$
\left\|\operatorname{dev} \boldsymbol{\sigma}^{(n+1)}\right\|=\left\|\operatorname{dev} \boldsymbol{\sigma}_{\text {trial }}^{(n+1)}\right\|-\Delta \gamma^{(n+1)} \rho 2 G \sqrt{\frac{3}{2}}
$$

which requires that:

$$
\Delta \gamma^{(n+1)} \leq \frac{\left\|\operatorname{dev} \boldsymbol{\sigma}_{\text {trial }}^{(n+1)}\right\|}{\rho 2 G \sqrt{\frac{3}{2}}}
$$


If this condition is verified, then the return mapping is made through the standard procedure described previously. Otherwise, the return mapping will be made to the apex of the DruckerPrager cone.

The stress at the apex point is:

$$
\boldsymbol{\sigma}_{\text {apex }}=p_{\min } \mathbf{1}=a \frac{\rho}{(1-\rho)}\left(\sigma_{y}-q\right) \mathbf{1}
$$

And, given that

$$
\boldsymbol{\sigma}_{\text {apex }}=\boldsymbol{\sigma}_{\text {trial }}^{(n+1)}-\mathbf{C}_{o}: \Delta \varepsilon_{p}^{(n+1)}
$$

the discrete increment of plastic strain is:

$$
\begin{aligned}
\Delta \varepsilon_{p}^{(n+1)} & =\mathbf{C}_{o}^{-1}:\left(\boldsymbol{\sigma}_{\text {trial }}^{(n+1)}-\boldsymbol{\sigma}_{\text {apex }}\right) \\
& =\frac{a}{3 K}\left(p_{\text {trial }}^{(n+1)}-p_{\text {min }}\right) \mathbf{1}+\frac{1}{2 G} \operatorname{dev} \boldsymbol{\sigma}_{\text {trial }}^{(n+1)}
\end{aligned}
$$

Notice that the value of $p_{\min }$ depends on the value of the isotropic hardening $q=q(\xi)$. Consequently, an iterative procedure is necessary in order to evaluate correctly the plastic multiplier.

Once the stress state arrives at the vertex of the cone, it will remain at the apex unless unloading or neutral loading occurs. This means that once the apex is reached, the consistent constitutive tensor is the null fourth order tensor.

\subsection{Softening behavior}

Physically, the energy dissipated during the formation of a slip surface is linked with the fracture energy $G_{f}$, defined by unit surface. When using a plastic model defined in terms of stress and strain to represent the behavior of the (regularized) slip surface, the dissipated plastic energy $W_{p}$ is defined by unit volume. In the discrete FE setting, these two definitions are related through a characteristic length $l_{c h}$, connected to the mesh resolution:

$$
W_{p}=\frac{G_{f}}{l_{c h}}
$$

In the plastic model, the rate of plastic work is computed as:

$$
\dot{W}_{p}=\boldsymbol{\sigma}: \dot{\varepsilon}_{p}=\bar{\sigma} \dot{\bar{\varepsilon}}_{p}
$$

where $\bar{\sigma}$ is the equivalent Drucker-Prager stress:

$$
\bar{\sigma}=\rho\left(\boldsymbol{\sigma}_{y}-q(\xi)\right)
$$

and $\dot{\bar{\varepsilon}}_{p}$ is the rate of equivalent plastic strain:

$$
\dot{\bar{\varepsilon}}_{p}=\sqrt{\frac{2}{3}}\left\|\dot{\varepsilon}_{p}\right\|=\left[\rho+a \sqrt{\frac{2}{3}}(1-\rho)\right] \dot{\xi}=\frac{\alpha}{\rho} \dot{\xi}
$$


where $\alpha \geq 1$ depends only on the friction angle. In both the linear and exponential softening cases, where $q(\xi)$ is defined by (39) and (40), respectively, the total plastic work is integrated to be:

$$
W_{p}=\int_{0}^{\infty} \dot{W}_{p} d t=\int_{0}^{\infty} \bar{\sigma} \dot{\bar{\varepsilon}}_{p} d t=\alpha \frac{\sigma_{y}^{2}}{2 H}
$$

From expressions (64) and (68), the parameter $H$ can be computed as:

$$
H=\alpha \frac{\sigma_{y}^{2}}{2 G_{f}} l_{c h}=\bar{H} l_{c h}
$$

The parameter $\bar{H}$ depends only on material properties, whereas $l_{c h}$ depends on the resolution of the discretization. As pointed out in Part II of this work [2], the size of the strain concentration band depends on the finite element technology. For instance, irreducible finite elements provide a concentration band within a single element span, due to the discontinuous strain field. On the contrary, in the $\varepsilon / u$ mixed FE formulation, with inter-elemental continuous strain, the slip line spans two elements. In this work, we will assume a locally computed $l_{\text {ch }}=2 h$, where $h$ is the representative size of the finite element, computed as $h_{e}^{2}=2 A_{e}$ for triangular elements, $h_{e}^{2}=A_{e}$ for quadrilateral elements and analogously in $3 \mathrm{D}$.

\subsection{Orientation of the shear band discontinuities}

Several authors $[22,23,24,25,26,27,28]$ have found analytical and geometrical solutions for the orientation of the discontinuity bands resulting from elasto-plastic models using different strategies. All of them seek their solutions after the so-called localization condition, which implies the loss of material ellipticity of the constitutive relation and is shown to be a necessary condition for the appearance of weak discontinuities and localized failure to take place.

In this work, a different approach is adopted to find analytical expressions for the orientation of localization bands for the Drucker-Prager model, both under plane strain and plane stress conditions. This procedure, proposed in reference [29] and exploited in reference [30], produces more realistic results than those used beforehand. It makes use of the stress boundedness and decohesion conditions, which, combined, can be shown to be necessary conditions for the shear band to form, and more constrictive than the localization condition. In fact, they can be shown to be necessary conditions for the occurrence of bifurcation and localization of the strain field, with bounded stresses and decohesion in the limit case along a localization band (or a regularized strong discontinuity). This is why the term strong discontinuity condition was used in reference [28] for it. However, it applies to localization bands (limited by weak discontinuities) and strong discontinuities alike.

The physical interpretation of this condition is simple: all of the difference in the strain field between the interior and the exterior points of the localization band, that is, the strain "jump", must be inelastic (plastic in this case). For a given plastic flow tensor, the condition may be used to determine the orientation of the discontinuity.

A remarkable difference between this approach and those other mentioned (based on the acoustic tensor) is that the orientation of the discontinuity does not depend on the elastic properties. It depends only on the plastic yield surface adopted and the stress state of interest.

In the next Section it is shown that this strategy predicts analytically orientations for the shear bands that are almost in perfect agreement with the ones computed numerically using the proposed stabilized mixed $\varepsilon / \mathbf{u}$ formulation. 


\section{$5 \quad$ Numerical examples}

The formulation presented in the preceding sections is illustrated below in a number of benchmark problems. Performance of the proposed stabilized mixed formulations is tested considering both 2D and $3 \mathrm{D}$ examples to demonstrate the generality of the formulation and its independence from the type of finite elements utilized. In 2D, plane-strain 3-noded linear triangular unstructured meshes are used. In 3D, structured meshes of regular triangular prisms are employed. The examples involve both compressible and incompressible plasticity using the Drucker-Prager model with exponential softening. Results obtained for the incompressible cases are compared with those obtained with the previously developed stabilized mixed pressure/displacement formulation $([4,5,6,7,8]$ and $[9])$.

The following material properties are assumed: Young's modulus $E=10 \mathrm{MPa}$, Poisson's ratio $\nu=0.3$, deviatoric stress threshold $\sigma_{y}=10 \mathrm{KPa}$ and fracture energy $G_{f}=400 \mathrm{~J} / \mathrm{m}^{2}$. For the Drucker-Prager model $a=1$, the cone is open in the triaxial compression end.

Values $c_{\varepsilon}=0.01$ and $c_{u}=1.0$ and $L=1 \mathrm{~m}$ are taken for the evaluation of the stabilization parameters. It is noted that the values adopted for the stabilization parameters do not affect the convergence rate of the formulation, which with the expression given by (20) is optimal for equal order interpolation [14]. For the mixed $\varepsilon / \mathbf{u}$ formulation, modified OSGS stabilization retaining only the volumetric-volumetric term is used. Using the other stabilization methods discussed in the paper produces similar results.

The Newton-Raphson method is used to solve the non-linear system of equations arising from the spatial and temporal discretization of the weak form of the stabilized problem. In all cases 200 equal time steps are performed to complete the analyses. Convergence of a step is attained when the ratio between the norms of the iterative residual forces and the incremental total forces is lower than $10^{-5}$. Calculations are performed with an enhanced version of the finite element program COMET [31, 32], developed by the authors at the International Center for Numerical Methods in Engineering (CIMNE). Pre and post-processing is done with GiD, also developed at CIMNE [33].

\subsection{Singly perforated strip}

The first example is a plane-strain singly perforated strip subjected to axial imposed straining. Because of the double symmetry of the domain and boundary conditions, only one quarter of the domain (the top right quarter) needs to be discretized. Figure 2a depicts the original geometry of the problem; dimensions are $20 \times 40 \mathrm{~m} \times \mathrm{m}$ (width $\times$ height) and the radius of the perforation is $r=1 \mathrm{~m}$. Thickness is $1 \mathrm{~m}$. A uniform upward vertical displacement is imposed at the top boundary.

The computational domain is divided into an unstructured uniform mesh of 7,202 linear triangles (3,721 nodes) with an average mesh size of $h=0.25 \mathrm{~m}$, not shown. The pre-processor used tends to introduce patches of equilateral triangles with predominant directions at $-30^{\circ},+30^{\circ}$ and $+90^{\circ}$ with the horizontal axis.

First, the incompressible case, with friction angle $\phi=0^{\circ}$ is investigated.

Figure 3 shows the results obtained using the stabilized mixed $\mathbf{u} / p$ formulation, once the shear bands are fully developed and the collapse mechanism can be appreciated ((half)-imposed vertical upward displacement $\delta=0.20 \mathrm{~m}$ ). The failure mechanism is correctly predicted, with $X$-shaped shear bands forming at $45^{\circ}$. No mesh-bias dependency is observed. The resolution of the shear 


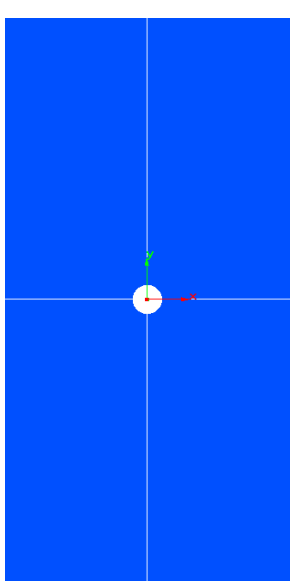

(a)

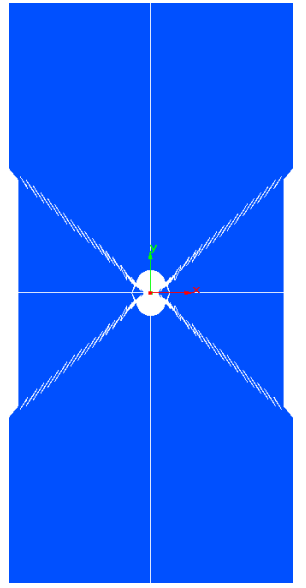

(b)

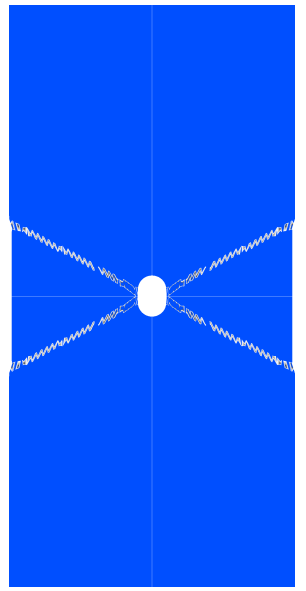

(c)

Figure 2: Geometries for the singly perforated strip: (a) undeformed, (b) deformed (x 5) $\phi=0^{\circ}$, (c) deformed (x 5) $\phi=45^{\circ}$

bands is optimal for the mesh used, as shown by the displacement and equivalent plastic strain plots. Discontinuity of the displacement tangential to the slip line and localization of the deviatoric strain occurs across one single element. The isochoric nature of the deformation pattern is demonstrated by the absence of volumetric plastic strains. No indication of overshoots or undershoots of any magnitude is observed at either side of the discontinuity lines. Control on the pressure is completely attained, and no spurious oscillations are observed anywhere in the domain.

Figure 4 shows the results obtained using the proposed stabilized mixed $\boldsymbol{\varepsilon} / \mathbf{u}$ formulation, also for a (half)-imposed vertical displacement $\delta=0.20 \mathrm{~m}$. Results are very similar to those obtained with the $\mathbf{u} / p$ formulation. The failure mechanism is correctly predicted and no mesh-bias dependency is observed. The resolution of the shear bands is also optimal for the mesh used. Now localization of the deviatoric strain occurs across two elements, because of inter-element strain continuity. Volumetric plastic strains are negligible. No spurious oscillation of any variable is observed. The deformed shape of the strip (with an amplification factor of 5) is shown in Figure 2.

Figure 5a compares (half)-load vs (half)-imposed vertical displacement curves (recall $1 \mathrm{~m}$ thickness is assumed) obtained with the two stabilized mixed formulations: $\mathbf{u} / p$ and $\varepsilon / \mathbf{u}$. Both mixed formulations capture adequately the peak load and the softening branch of the curve, but the response obtained with the newly proposed formulation is less dissipative. The reason for this is that this formulation is locally more accurate and it reduces the stress locking induced by the isochoric deformation behavior inside the shear bands.

The total dissipated energy required to create a perfectly straight shear band branch at $45^{\circ}$, similar to those shown in Figures 3 and 4 , but without any boundary effect, is $W_{d i s}=G_{f} \cdot A=$ $400 \cdot 9 \sqrt{2} \cdot 1=5091 \mathrm{~J}$. The work spent by the external forces in the $\varepsilon / \mathbf{u}$ formulation (area under the curve in Figure 5a) is $W_{d i s}^{\varepsilon / \mathbf{u}}=5210 \mathrm{~J}$ (2.33\% difference with respect the idealized solution), while the work spent by the $\mathbf{u} / p$ formulation is $W_{d i s}^{\mathbf{u} / p}=6723 \mathrm{~J}$ (32.02\% difference). The accuracy of the proposed formulation is remarkable. 


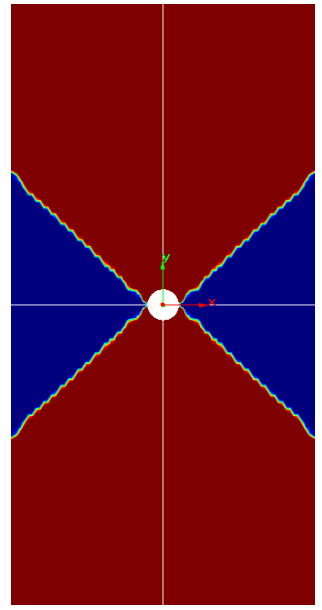

(a)

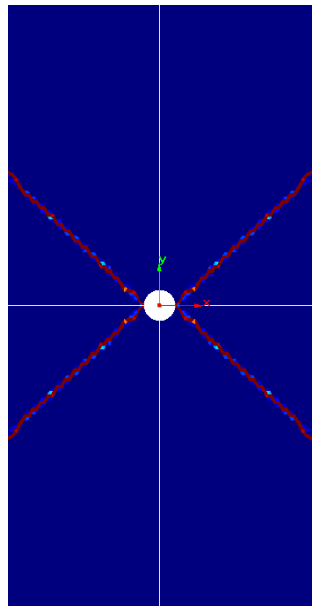

(b)

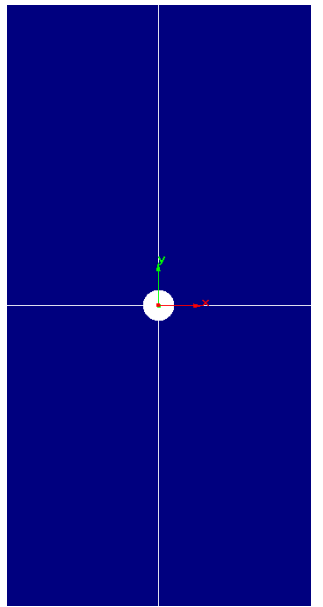

(c)

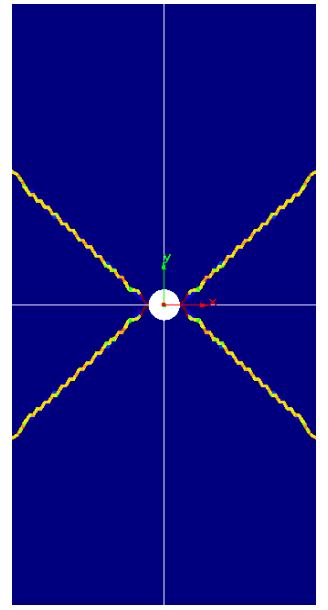

(d)

Figure 3: Results for singly perforated strip using the mixed $\mathbf{u} / p$ formulation; incompressible case $\phi=0^{\circ}$. Contours for: (a) vertical displacement, (b) equivalent plastic strain, (c) volumetric plastic strain and (d) deviatoric plastic strain

Figure 5a also shows the (half)-load vs (half)-imposed vertical displacement curves obtained with the two stabilized mixed formulations, $\mathbf{u} / p$ and $\varepsilon / \mathbf{u}$, on a refined unstructured uniform mesh of 20,255 linear triangles (10,342 nodes) with an average mesh size of $h=0.15 \mathrm{~m}$. These show that the solution obtained with $\varepsilon / \mathbf{u}$ formulation is independent of the mesh size and bias. Contrarily,

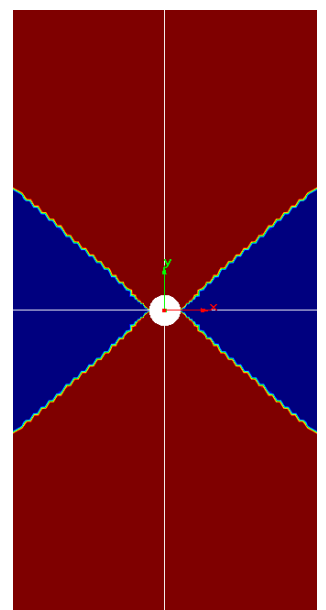

(a)

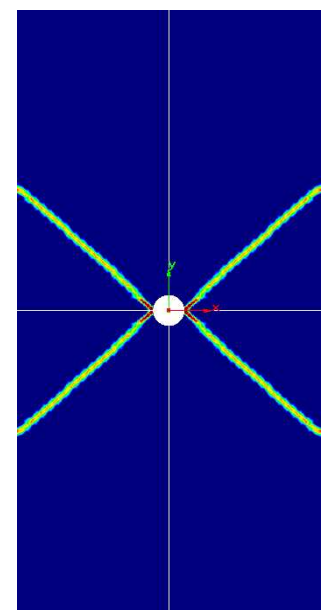

(b)

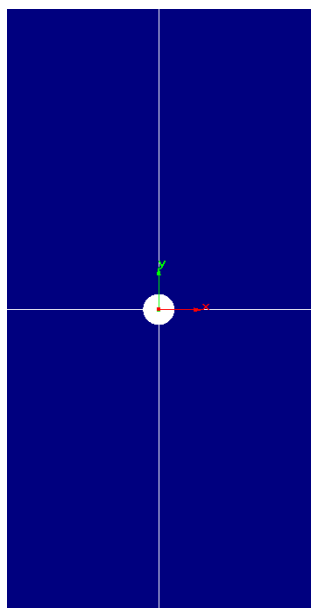

(c)

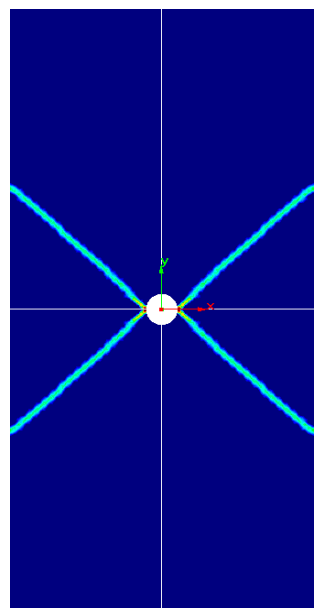

(d)

Figure 4: Results for singly perforated strip with the mixed $\varepsilon / \mathbf{u}$ formulation; incompressible case $\phi=0^{\circ}$. Contours for: (a) vertical displacement, (b) equivalent plastic strain, (c) volumetric plastic strain and (d) deviatoric plastic strain 


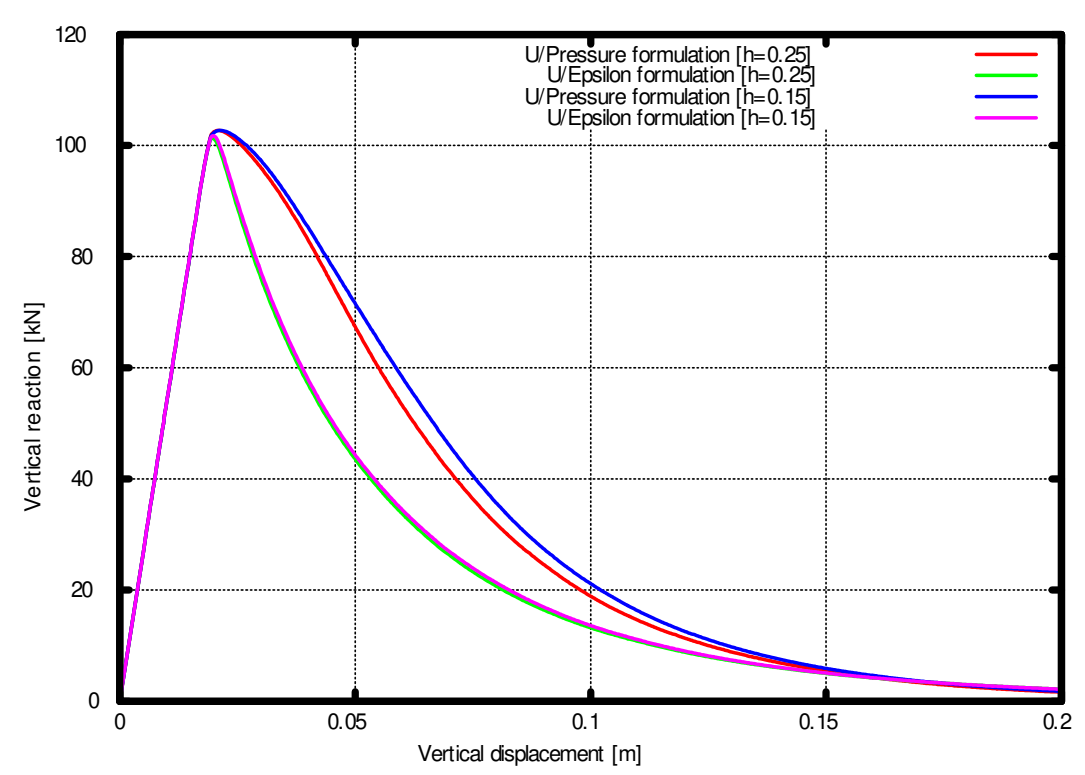

(a)

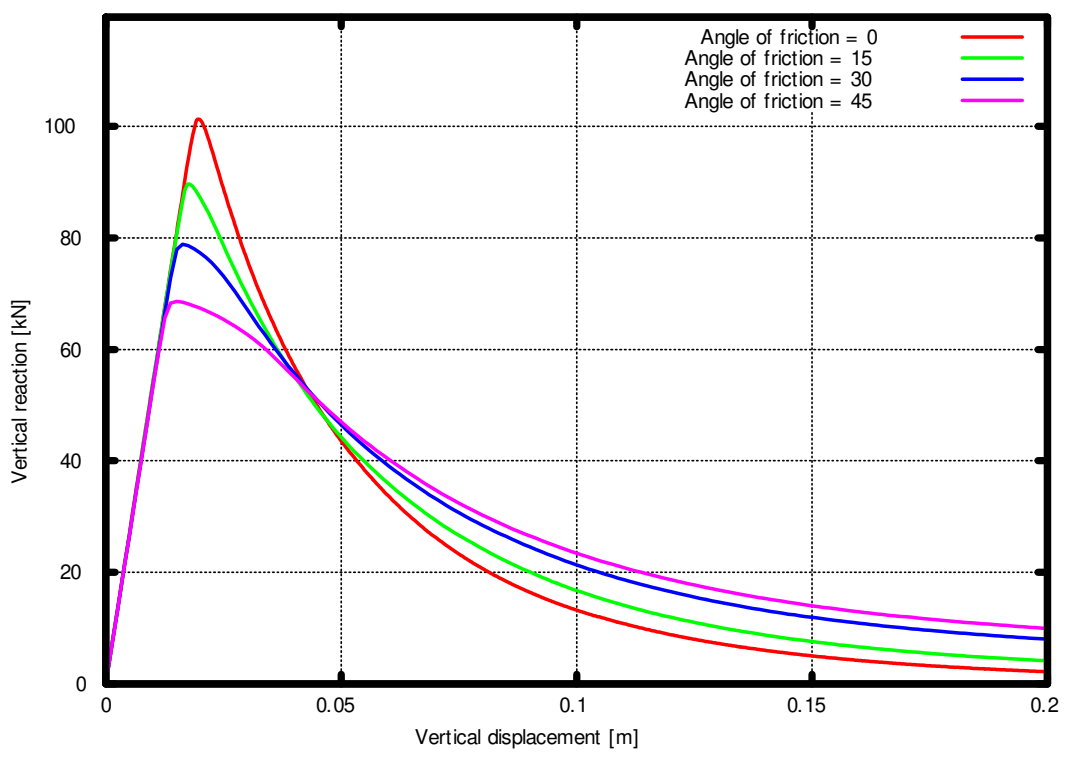

(b)

Figure 5: Results for singly perforated strip. (a) Comparison between the mixed $\mathbf{u} / p$ and $\varepsilon / \mathbf{u}$ formulations for the incompresible case. Effect of mesh refinement. (b) Comparison for the $\varepsilon / \mathbf{u}$ formulation for different friction angles. 
the $\mathbf{u} / p$ formulation converges to an over-dissipative solution. This is due to the stress-locking induced by the poor kinematics of the elements used under localized shear. The problem is much alleviated in the $\varepsilon / \mathbf{u}$ formulation (see reference [2]).

Next, compressible cases, with increasing friction angles $\phi=15^{\circ}, 30^{\circ}, 45^{\circ}$ are investigated.

Figure 5b shows (half)-load vs (half)-imposed vertical displacement curves obtained with the $\varepsilon / \mathbf{u}$ formulation for these cases. The limit load reduces as the friction angle increases, due to the orientation of the cone $(a=1)$. Even if the dissipated energy per unit area remains constant, and equal for the fracture energy of the material, the total dissipated energy decreases as the friction angle increases, because the length of the shear band diminishes.

Table 1 shows the comparison between the analytical localization angles computed for uniaxial tension in plane strain conditions and the ones obtained numerically. The analytical values are obtained with the procedure presented in references [29] and [30]. The remarkable agreement between the analytical and the numerical values validates both the analytical and the numerical approaches.

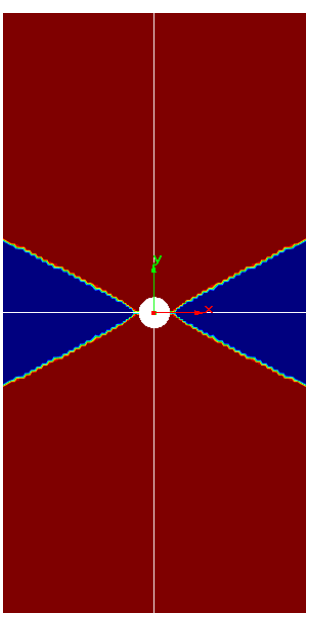

(a)

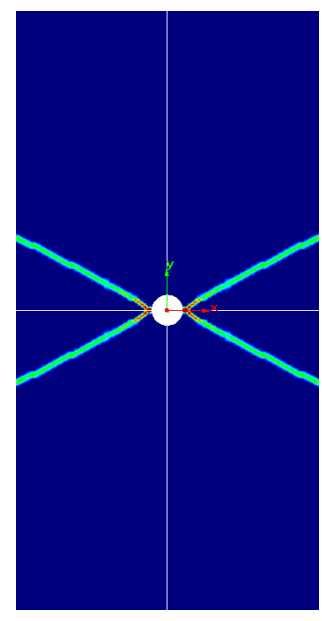

(b)

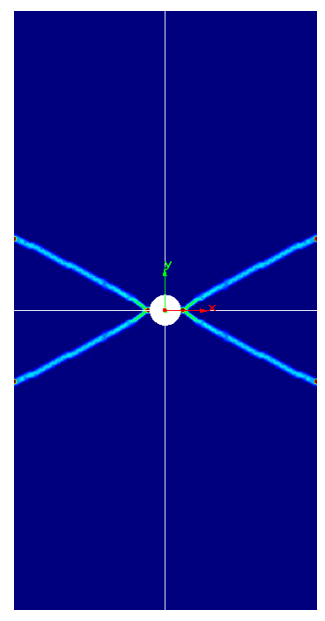

(c)

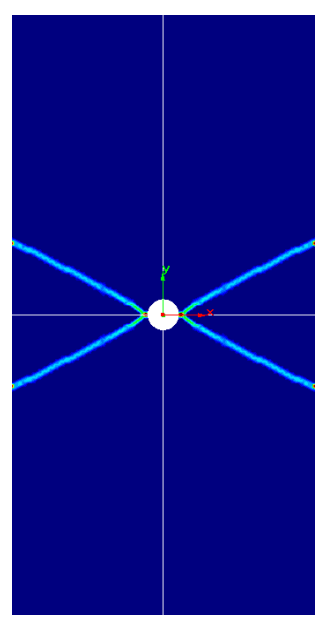

(d)

Figure 6: Results for singly perforated strip with the mixed $\varepsilon / \mathbf{u}$ formulation; compressible case $\phi=45^{\circ}$. Contours for: (a) vertical displacement, (b) equivalent plastic strain, (c) volumetric plastic strain and $(\mathrm{d})$ deviatoric plastic strain

\begin{tabular}{|c|c|c|}
\hline Plane strain & Analytical [29], [30] & Numerical \\
\hline$\phi=0^{\circ}$ & $\theta_{l o c}=45.00^{\circ}$ & $\theta_{l o c}=44.32^{\circ}$ \\
\hline$\phi=15^{\circ}$ & $\theta_{l o c}=40.53^{\circ}$ & $\theta_{l o c}=39.30^{\circ}$ \\
\hline$\phi=30^{\circ}$ & $\theta_{l o c}=35.07^{\circ}$ & $\theta_{l o c}=33.90^{\circ}$ \\
\hline$\phi=45^{\circ}$ & $\theta_{l o c}=26.12^{\circ}$ & $\theta_{l o c}=26.90^{\circ}$ \\
\hline
\end{tabular}

Table 1: Results for singly perforated strip with the mixed $\varepsilon / \mathbf{u}$ formulation. Comparison between the analytical localization angles for uniaxial tension in plane strain conditions and the ones obtained numerically 
Figure 6 shows the results obtained using the proposed stabilized mixed $\varepsilon / \mathbf{u}$ formulation, friction angle $\phi=45^{\circ}$, and a (half)-imposed vertical displacement $\delta=0.20 \mathrm{~m}$. The failure mechanism is correctly predicted, with shear band now forming an angle of $26.90^{\circ}$ with the horizontal axis (being $26.11^{\circ}$ the analytical value), completely independent of the mesh-bias. The resolution of the shear bands is also optimal for the mesh used. Volumetric plastic strains are of the same order than the deviatoric plastic strains. The deformed shape (x 5) of the strip is shown in Figure 2c.

\subsection{Circular rigid inclusion}

The second example is a plane-strain circular rigid inclusion subjected to an imposed vertical downward imposed displacement. Perfect stick conditions are assumed between the inclusion and the surrounding medium; thus, the vertical displacement is imposed directly to the interface. Figure 7a depicts the original geometry of the problem; dimensions are $20 \times 20 \mathrm{~m} \times \mathrm{m}$ (width $\times$ height) and the radius of the inclusion is $r=1 \mathrm{~m}$. Thickness is $1 \mathrm{~m}$.

This example is interesting because the symmetric collapse mechanism consists of two almost circular curved shear bands that intersect each other. Therefore, it is an adequate test to assess the ability of the different formulations to deal with such a complex situation in a given mesh.

Because of the symmetry of the domain and boundary conditions with respect the central vertical axis, only one half of the domain (the right half) needs to be discretized. The computational domain is divided into an unstructured non-uniform mesh of 13,750 linear triangles (7,006 nodes) with smaller elements near the circular inclusion, not shown.

The incompressible case, with friction angle $\phi=0^{\circ}$ is investigated.

Figure 8 shows the results obtained using the stabilized mixed $\mathbf{u} / p$ formulation, once the collapse mechanism and the shear bands are fully developed. The failure mechanism, which can be appreciated in Figure 8a, displaying the contour fills for the norm of the displacements, and Figure $8 \mathrm{~b}$, displaying the contour fills for the norm of the equivalent plastic strain, is correctly predicted. Because of the formulation used, discontinuity of the displacements across the slip lines and localization of the plastic strain occurs across one single element. The attained resolution is optimal

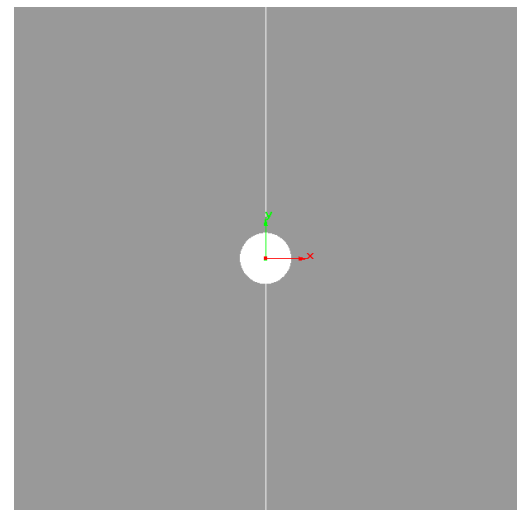

(a)

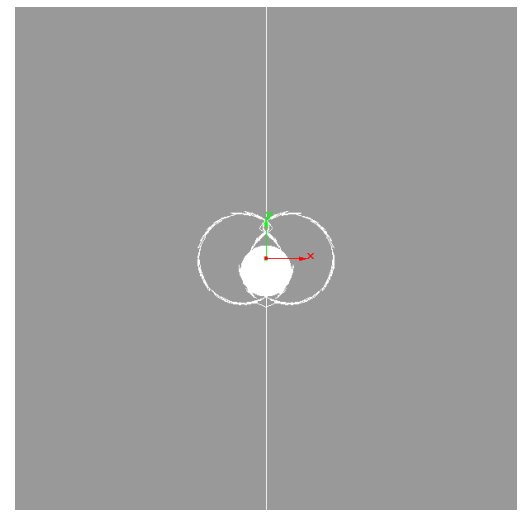

(b)

Figure 7: Original and deformed (x 5) geometries for circular rigid inclusion 


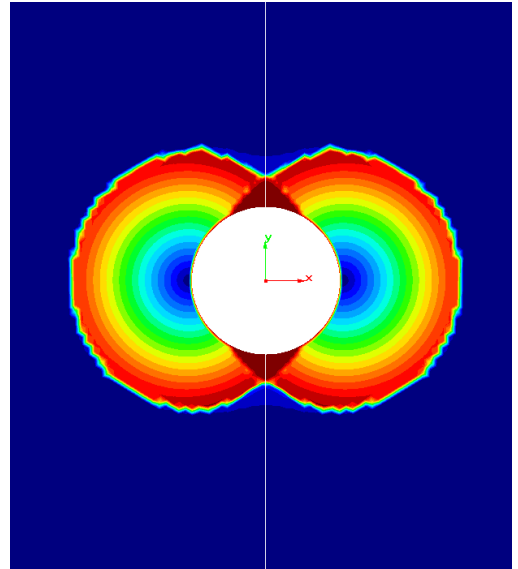

(a)

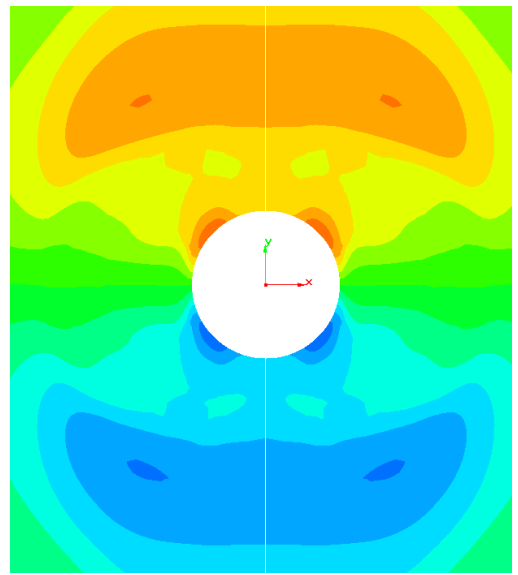

(c)

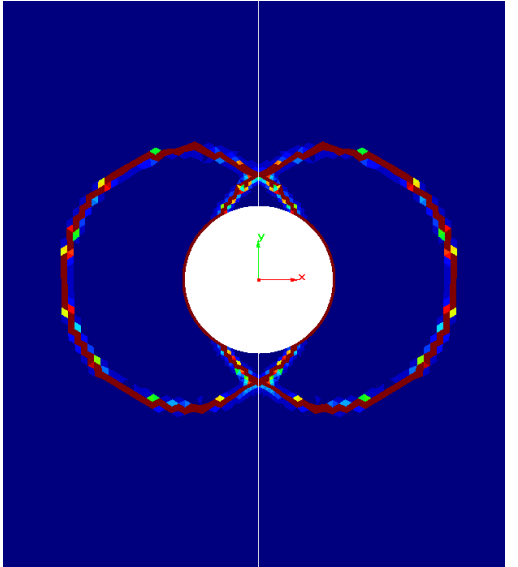

(b)

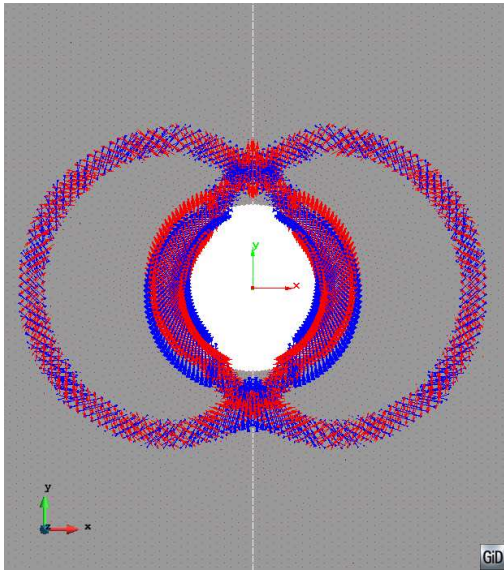

(d)

Figure 8: Results for circular rigid inclusion using the mixed $\mathbf{u} / p$ formulation. Incompressible plasticity, $\phi=0^{\circ}$. Contours for: (a) displacement, (b) equivalent plastic strain, (c) pressure and (d) principal strain vectors

for the mesh used. Figure 8c shows pressure contours. Not only there is no evidence of pressure oscillations, but the pressure field is completely undisturbed by the presence of the shear bands. An almost perfectly skew-symmetric pressure distribution is attained. Finally, Figure 8d shows the principal strain vectors at failure. Strain localization is clear, and the direction of the computed vectors is affected by the mesh alignment, although this is not evident because the mesh is unstructured. The deformed shape of the problem (with a displacement amplification factor of 5 ) is shown in Figure 7.

Figure 9 shows the corresponding results obtained using the proposed stabilized mixed $\varepsilon / \mathbf{u}$ formulation. As in the previous example, results are qualitatively very similar to those obtained with the $\mathbf{u} / p$ formulation. The failure mechanism is obviously the same and no mesh-bias dependency is 


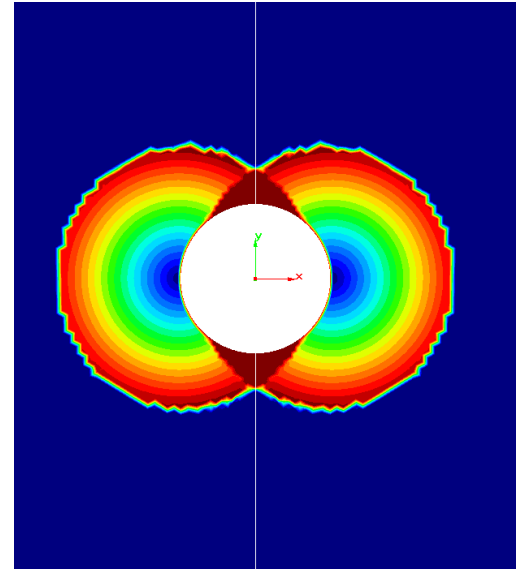

(a)

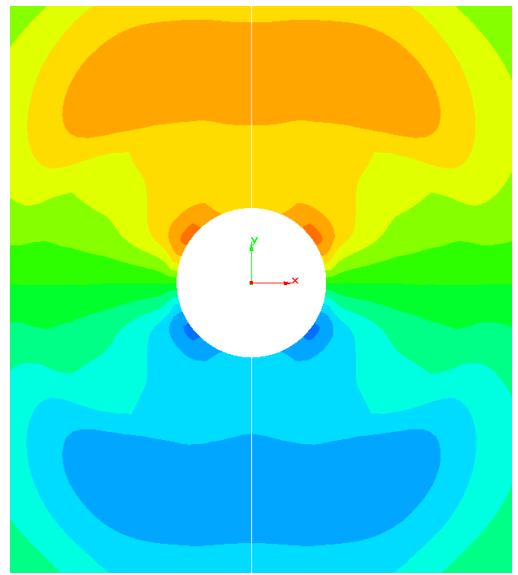

(c)

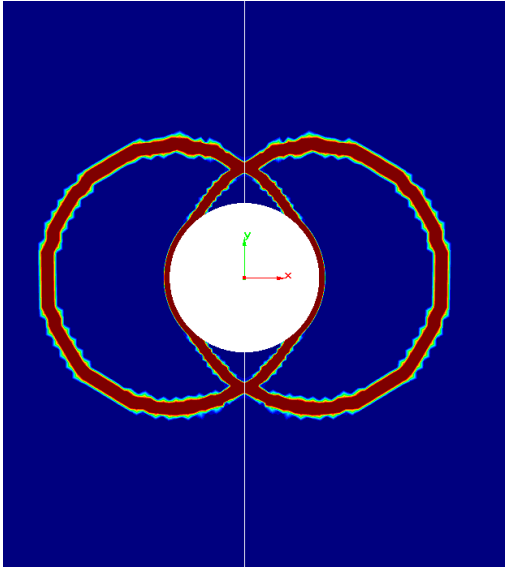

(b)

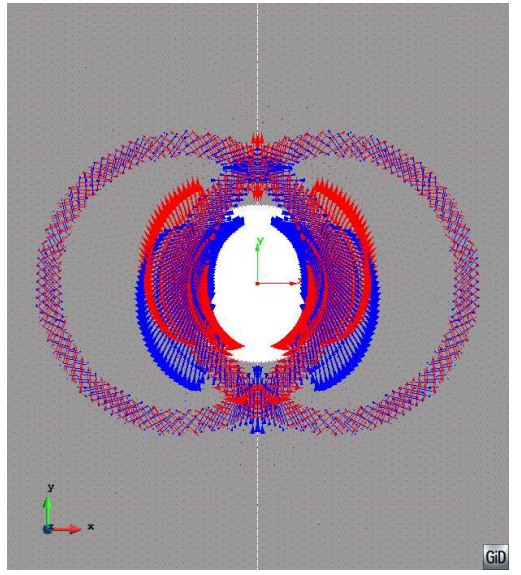

(d)

Figure 9: Results for circular rigid inclusion using the mixed $\varepsilon / \mathbf{u}$ formulation. Incompressible plasticity, $\phi=0^{\circ}$. Contours for: (a) displacement, (b) equivalent plastic strain, (c) pressure and (d) principal strain vectors

observed. Contour plots for the displacement and the pressure fields, Figures 9a and 9c are almost identical to those in $8 \mathrm{a}$ and $8 \mathrm{c}$, because in both formulations these fields are linearly interpolated. A clear difference can be observed in the contour plot of the plastic strain, Figure 9b, which can be considered as the smoothing of the piece-wise discontinuous field of Figure 8c. The inter-element continuity of the plastic strain is clear in the picture. Figure $9 \mathrm{~d}$ shows the principal strain vectors at failure. In this case, the direction of the computed vectors is much less affected by the mesh alignment than in Figure 8d.

Figure 10 compares (half)-load vs imposed vertical displacement curves obtained with the two stabilized mixed formulations: $\boldsymbol{u} / p$ and $\boldsymbol{\varepsilon} / \mathbf{u}$. As in the previous example, both mixed formulations capture well the limit load and the general softening trend of the curve, but the response obtained 


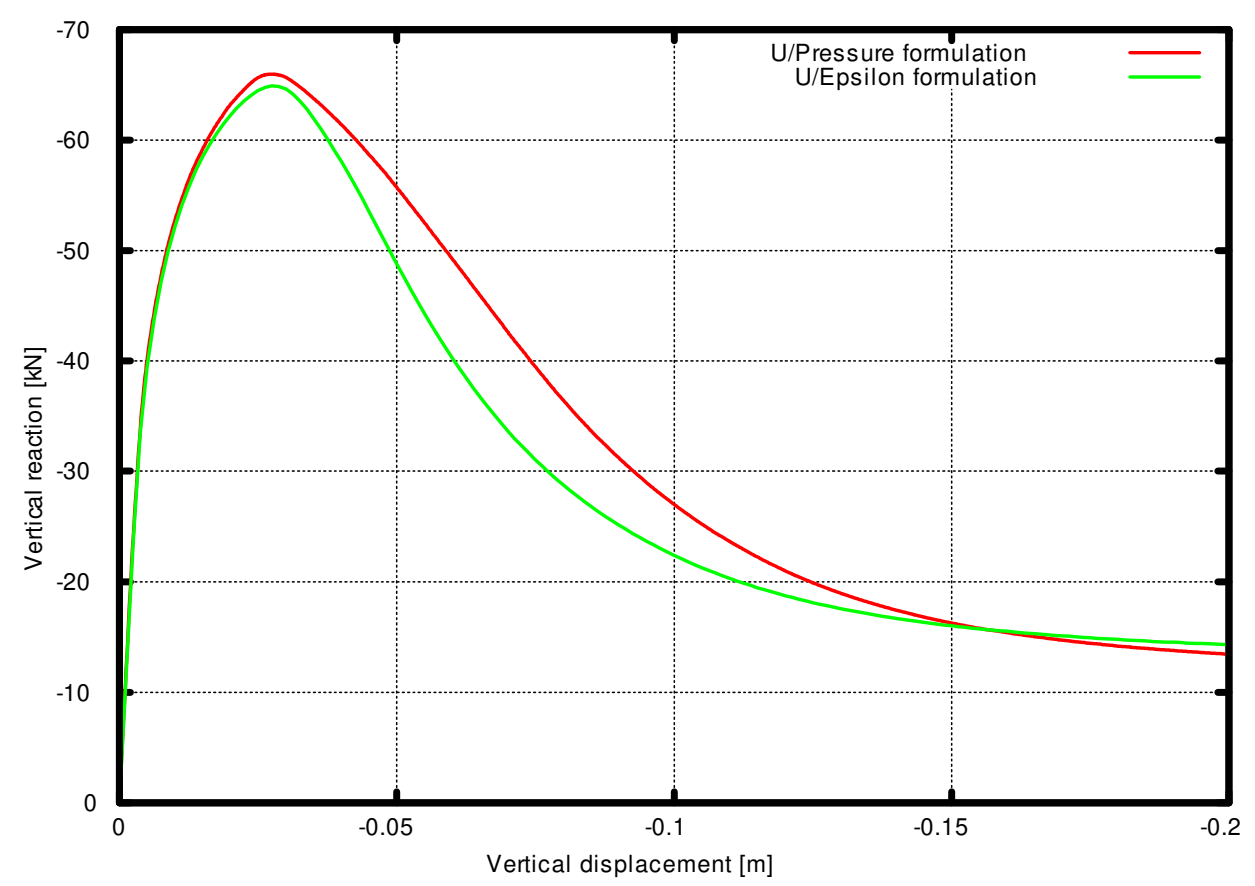

Figure 10: Force vs. displacement plot for circular rigid inclusion. Comparison between the $\mathbf{u} / p$ and the $\varepsilon / \mathbf{u}$ formulations

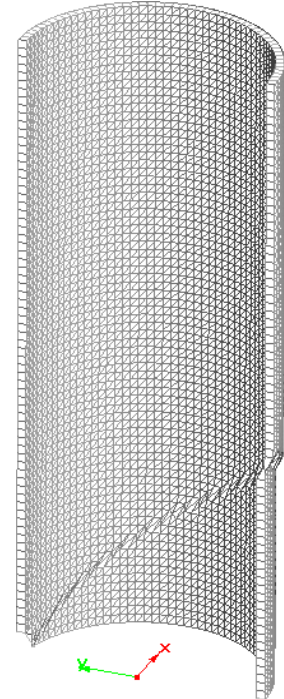

(a)

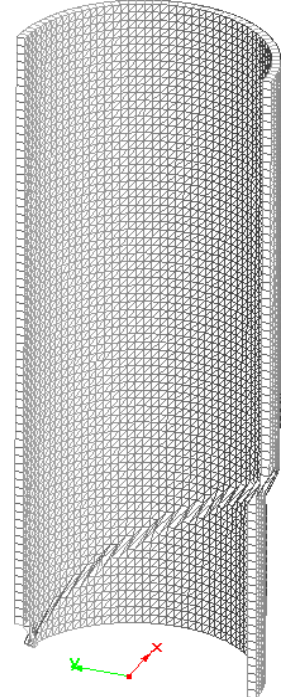

(b)

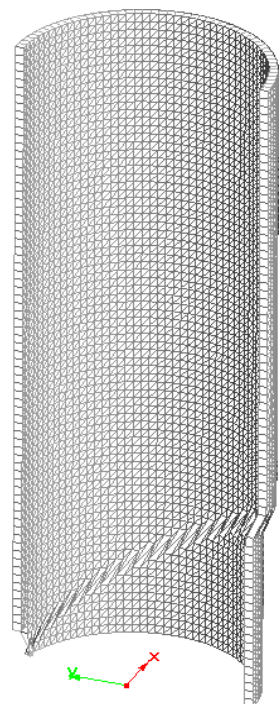

(c)

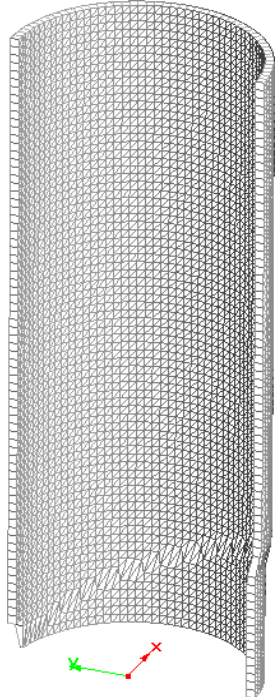

(d)

Figure 11: Deformed (x 5) geometries for singly perforated thin-walled cylinder for different friction angles: (a) $0^{\circ}$, (b) $15^{\circ}$, (c) $30^{\circ}$, (d) $45^{\circ}$

with the newly proposed formulation is more accurate and less dissipative. 


\subsection{Simply perforated thin-walled cylinder}

The last example is a simply perforated thin-walled cylinder subjected to axial imposed straining. Dimensions of the cylinder are: height $30 \mathrm{~m}$, outer radius $6 \mathrm{~m}$, inner radius $5.8 \mathrm{~m}$, thickness 0.2 $\mathrm{m}$. The perforation is a square indentation of trace $0.4 \times 0.4 \mathrm{~m}^{2}$. Because of the double symmetry of the domain and boundary conditions, only one quarter of the cylinder is discretized. A uniform upward vertical displacement is imposed at the top boundary. Because the cylinder is thin-walled and there is no restriction to deformation in the radial and hoop directions, the normal stresses in the radial and hoop directions are null, so that the cylinder is subjected to a state of uniaxial vertical stress. Therefore, the angles of the localized shear bands can be analytically obtained under plane stress conditions.

The computational domain is divided into a structured uniform mesh of 3,749 triangular prisms (7,750 nodes) with a mesh size of $h=0.2 \mathrm{~m}$, half the size of the indentation and equal to the thickness of the cylinder.

Incompressible and compressible cases, with increasing friction angles $\phi=0^{\circ}, 15^{\circ}, 30^{\circ}, 45^{\circ}$ are investigated using the stabilized mixed $\varepsilon / \mathbf{u}$ formulation. The deformed shapes obtained are shown in Figure 11 (amplification factor 5). The failure mechanisms are correctly predicted in all cases, with $X$-shaped helicoidal shear bands forming at different orientations, independently of the marked alignment of the structured mesh, and without the use of any auxiliary tracking technique. It can be observed that, as in the first example, the angle that the shear band forms with the horizontal plane decreases as the angle of friction of the material increases.

Table 2 shows the comparison between the analytical localization angles computed for uniaxial tension in plane stress conditions and the ones obtained numerically. The analytical values are obtained with the procedure presented in references [29] and [30]. Note that the localization angles are different in plane stress situations than under plane strain conditions. As in the first example, the agreement between the analytical and the numerical values is remarkable, and validates both the analytical and the numerical approaches.

This example provides a case to illustrate not only the quantitative, but the qualitative difference between the $\boldsymbol{u} / p$ and $\boldsymbol{\varepsilon} / \mathbf{u}$ formulation. Figure 12 shows the results obtained for the incompressible case $\left(\phi=0^{\circ}\right)$, with the $\varepsilon / \mathbf{u}$ formulation. As can be noted, the computed failure mechanism is correct, as all plots, vertical displacement, equivalent plastic strain, pressure and principal strain vectors, corroborate. Figure 13 shows the results obtained with the $\boldsymbol{u} / p$ formulation. It can be appreciated that the solution obtained with this formulation is not realistic. Figure 13c shows that in this case the obtained plastic strain localizes in a layered pattern which is discontinuous from one

\begin{tabular}{|c|c|c|}
\hline Plane stress & Analytical [29], [30] & Numerical \\
\hline$\phi=0^{\circ}$ & $\theta_{l o c}=35.26^{\circ}$ & $\theta_{l o c}=34.04^{\circ}$ \\
\hline$\phi=15^{\circ}$ & $\theta_{l o c}=31.55^{\circ}$ & $\theta_{l o c}=31.20^{\circ}$ \\
\hline$\phi=30^{\circ}$ & $\theta_{l o c}=26.92^{\circ}$ & $\theta_{l o c}=26.07^{\circ}$ \\
\hline$\phi=45^{\circ}$ & $\theta_{l o c}=19.47^{\circ}$ & $\theta_{l o c}=20.44^{\circ}$ \\
\hline
\end{tabular}

Table 2: Results for simply perforated thin-walled cylinder with the mixed $\varepsilon / \mathbf{u}$ formulation. Comparison between the analytical localization angles for uniaxial tension in plane stress conditions and the ones obtained numerically 


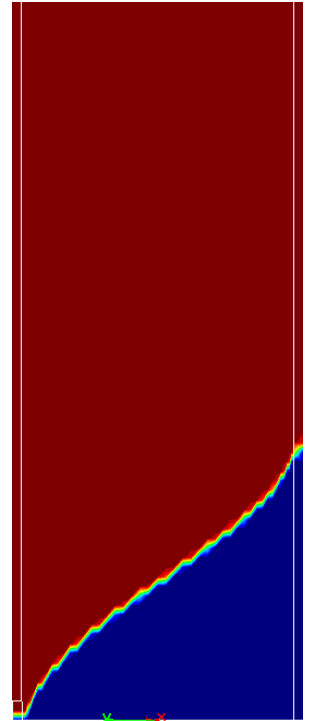

(a)

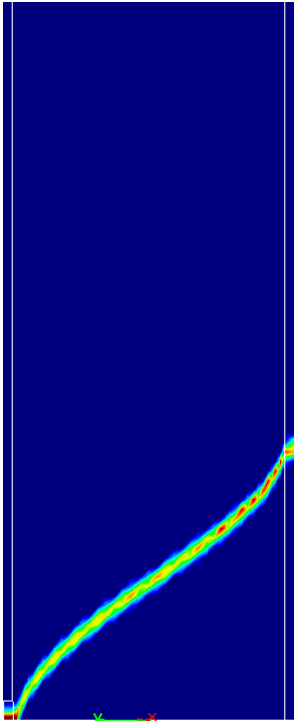

(b)

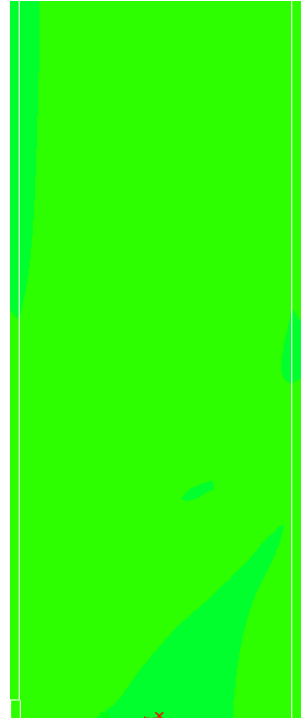

(c)

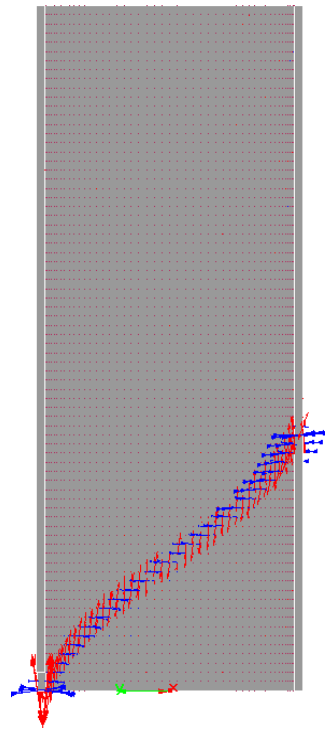

(d)

Figure 12: Results for simply perforated thin-walled cylinder with the mixed $\varepsilon / \mathbf{u}$ formulation. Contours for: (a) vertical displacement, (b) equivalent plastic strain, (c) pressure and (d) principal strain vectors

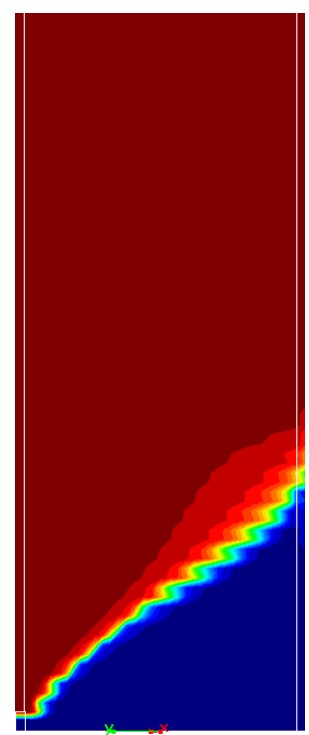

(a)

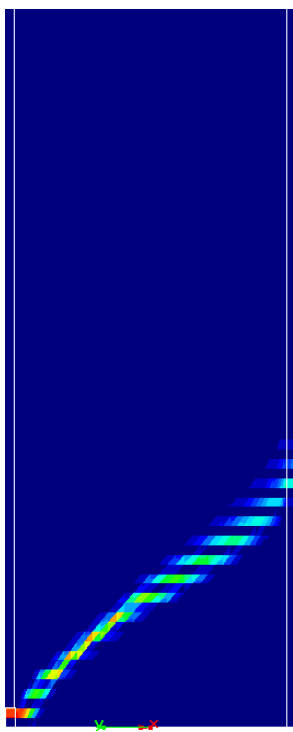

(b)

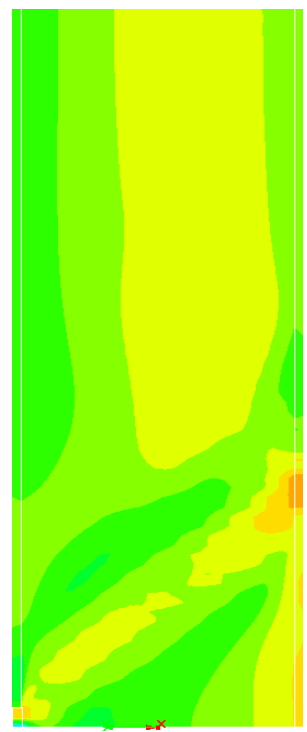

(c)

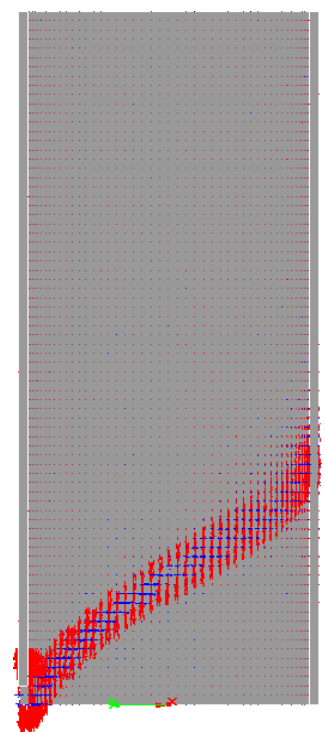

(d)

Figure 13: Results for simply perforated thin-walled cylinder with the mixed $\mathbf{u} / p$ formulation. Contours for: (a) vertical displacement, (b) equivalent plastic strain, (c) pressure and (d) principal strain vectors 


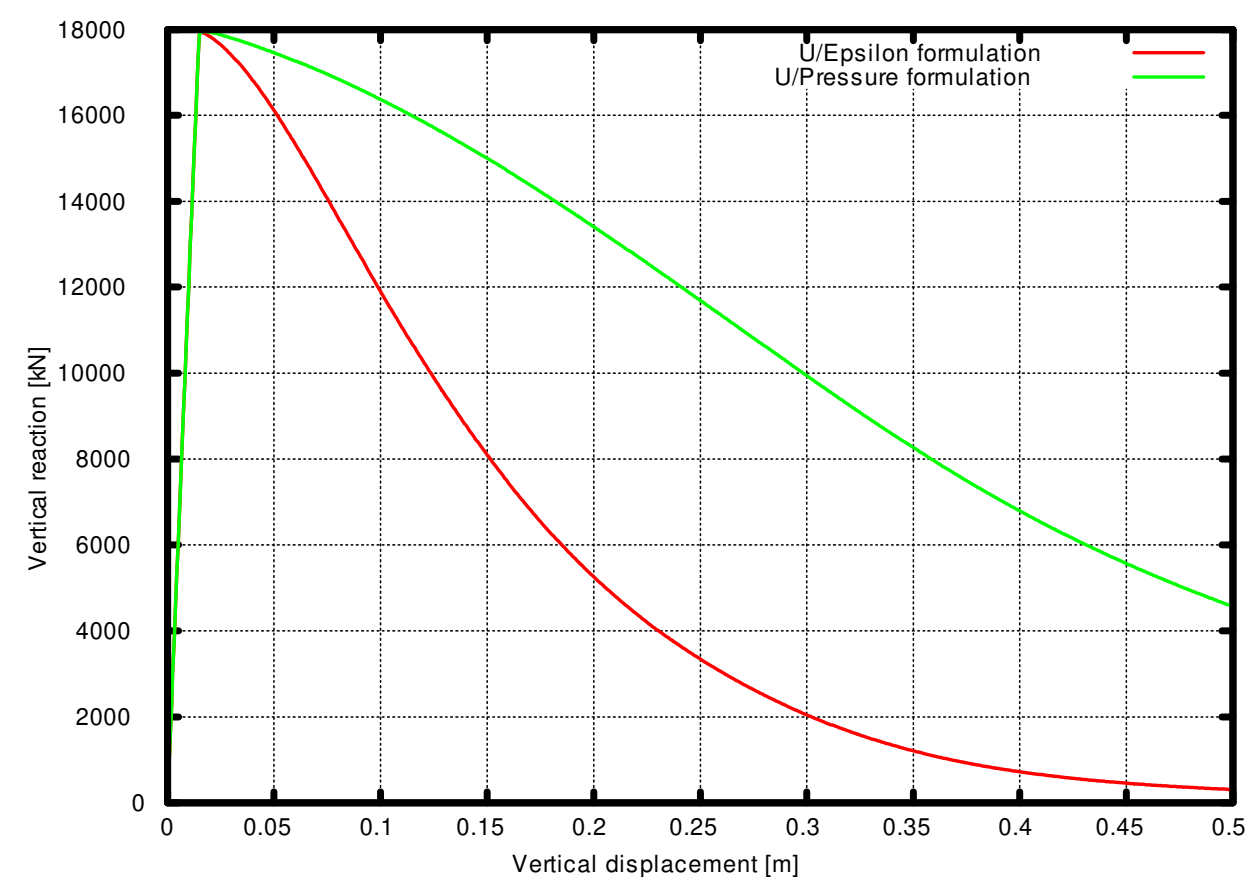

Figure 14: Force vs. displacement plot for simply perforated thin-walled cylinder. Comparison between the $\mathbf{u} / p$ and the $\varepsilon / \mathbf{u}$ formulations

horizontal layer of elements to the ones above or below. This spurious type of stepped localization is possible with the $\boldsymbol{u} / p$ discrete formulation, where the deviatoric strains are discontinuous, but cannot occur with the $\varepsilon / \mathbf{u}$ discrete formulation, where all strains are continuous. Note that the solution of the $\boldsymbol{u} / p$ discrete formulation cannot be improved by restoring to mesh refiment.

Figure 14 compares (half)-load vs (half)-imposed vertical displacement curves obtained with the two stabilized mixed formulations: $\boldsymbol{u} / p$ and $\boldsymbol{\varepsilon} / \mathbf{u}$. Here, the incorrect prediction of the failure mechanism when using the mixed $\boldsymbol{u} / p$ formulation shows in the over prediction of the dissipated energy spent during the failure process.

\section{Conclusions}

This paper presents the application of a stabilized mixed strain/displacement finite element formulation for the solution nonlinear solid mechanics problems involving compressible and incompressible plasticity. Such formulation presents two advantages when compared to the standard, displacement based, irreducible formulation: (a) it provides enhanced strain (and stress) rate of convergence and (b) it is able to deal with incompressible situations. The first advantage applies also to the mixed pressure/displacement formulation.

The variational multiscale stabilization introduced allows the use of equal order interpolations in a consistent way. Consequently, low order finite elements with continuous strain and displacement fields are used in conjunction with an associative frictional Drucker-Prager plastic model to model strain localization and failure. The derived model yields a general and robust scheme, suitable for 
engineering applications. Its application translates in the achievement of the goals:

1. the resulting discrete FE model is well posed and stable,

2. the formulation is able to tackle compressible and incompressible inelastic behavior, without indications of pressure oscillations in the latter case,

3. the formulation is able to tackle directional inelastic behavior, and

4. the results are convergent and not spuriously dependent of the finite element mesh used.

Benchmark numerical examples (using triangles in 2D and triangular prisms in 3D) show the substantial advantage of the mixed formulation over the irreducible and the mixed pressure/displacement one to predict correct failure mechanisms with localized patterns of strain, virtually free from any dependence of the mesh directional bias.

The proposed formulation has two salient features. On one side, it is of general application, in $2 \mathrm{D}$ and $3 \mathrm{D}$ problem, to structured and unstructured meshes and to simplicial or non simplicial elements. On the other side, no "ad hoc" auxiliary crack tracking technique is necessary.

\section{Acknowledgments}

Financial support from the EC 7th Framework Programme under the MuMoLaDe project - Multiscale Modelling of Landslides and Debris Flows - within the framework of Marie Curie ITN (Initial Training Networks, ref. 289911) and the Spanish Ministry of Economy and Competitiveness under the EACY project - Enhanced accuracy computational and experimental framework for strain localization and failure mechanisms- within the "Excellency" Programme for Knowledge Generation, ref. MAT2013-48624-C2-1-P, is gratefully acknowledged.

\section{References}

[1] Cervera, M., Chiumenti, M. and Codina, R. (2010). Mixed stabilized finite element methods in nonlinear solid mechanics. Part I: Formulation, Comp. Meth. in Appl. Mech. and Eng., 199, 2559-2570.

[2] Cervera, M., Chiumenti, M. and Codina, R. (2010). Mixed stabilized finite element methods in nonlinear solid mechanics. Part II: Strain localization, Comp. Meth. in Appl. Mech. and Eng., 199, 2571-2589.

[3] Cervera, M., Chiumenti, M. and Codina, R. (2011). Mesh objective modelling of cracks using continuous linear strain and displacement interpolations, Int. J. for Numerical Methods in Eng., 87, 962-987.

[4] Cervera, M., Chiumenti, M., Valverde, Q. and Agelet de Saracibar, C. (2003). Mixed Linear/linear Simplicial Elements for Incompressible Elasticity and Plasticity. Comp. Meth. in Appl. Mech. and Eng., 192, 5249-5263. 
[5] Cervera, M., Chiumenti, M. and Agelet de Saracibar, C. (2004). Softening, localization and stabilization: capture of discontinuous solutions in J2 plasticity. Int. J. for Num. and Anal. Meth. in Geomechanics, 28, 373-393.

[6] Cervera, M., Chiumenti, M. and Agelet de Saracibar, C. (2004). Shear band localization via local $\mathrm{J}_{2}$ continuum damage mechanics. Comp. Meth. in Appl. Mech. and Eng., 193, 849-880.

[7] Chiumenti, M., Valverde, Q., Agelet de Saracibar, C. and Cervera, M., (2004). A stabilized formulation for incompressible plasticity using linear triangles and tetrahedra, Int. J. of Plasticity, 20, 1487-1504.

[8] Agelet de Saracibar C., Chiumenti M., Valverde Q., and Cervera M. (2006). On the Orthogonal Subgrid Scale Pressure stabilization of Small and Finite deformation J2 Plasticity, Computer Methods in Applied Mechanics and Engineering, 195, 1224-1251.

[9] Cervera, M. and Chiumenti, M. (2009). Size effect and localization in $\mathrm{J}_{2}$ plasticity, International Journal of Solids and Structures 46, 3301-3312.

[10] Brezzi, F. and Fortin, M. (1991) Mixed and Hybrid Finite Element Methods, Spinger.

[11] Hughes, T.J.R., Feijoó, G.R., Mazzei. L., Quincy, J.B. (1998) . The variational multiscale method-a paradigm for computational mechanics, Comp. Meth. in Appl. Mech. and Eng. 166, $3-28$.

[12] Codina, R. (2000) . Stabilization of incompressibility and convection through orthogonal subscales in finite element methods, Comp. Meth. in Appl. Mech. and Eng. 190, 1579-1599.

[13] Codina, R. and Blasco, J. (1997) . A finite element method for the Stokes problem allowing equal velocity-pressure interpolations, Comp. Meth. in Appl. Mech. and Eng. 143, 373-391.

[14] Badia, S. and Codina, R. (2009) . Unified stabilized finite element formulations for the Stokes and the Darcy problems, SIAM Journal on Numerical Analysis 17, 309-330.

[15] Codina, R. (2008). Analysis of a stabilized finite element approximation of the Oseen equations using orthogonal subscales, Applied Numerical Mathematics, 58, 264-283.

[16] Castillo, E. and Codina, R. (2014). Variational multi-scale stabilized formulations for the stationary three-field incompressible viscoelastic flow problem, Comp. Meth. in Appl. Mech. and Eng., 279, 579-605.

[17] Simo, J.C. and Hughes, T.J.R. (1998). Computacional Inelasticity. Interdisciplinary Applied Mathematics. Vol. 7. Springer.

[18] Simo, J. C. and Taylor, R. L. (1985). Consistent tangent operators for rate-independent elastoplasticity, Comp. Meth. in Appl. Mech. and Eng. 48, 101-118.

[19] deBorst, R. (1987). Integration of plasticity equations for singular yield functions, Computers \& Structures 26, 823 - 829 . 
[20] Crisfield, M. (1997). Non-linear Finite Element Analysis of Solids and Structures. II: Advanced topics. Wiley.

[21] Peric, D. and de Souza Neto, E. A. (1999). A new computational model for Tresca plasticity at finite strains with an optimal parametrization in the principal space, Comp. Meth. in Appl. Mech. and Eng. 171, 463 - 489.

[22] Ottosen, N. and Runesson, K. (1991). Properties of discontinuous bifurcation solutions in elasto-plasticity. Int. J. Solids and Structures 27(4), 401-421.

[23] Runesson, K., Ottosen, N.S., and Peric, D. (1991). Discontinuous bifurcations of elastic-plastic solutions at phase stress and plane strain. Int. J. of Plasticity 7, 99-121.

[24] Steinmann, P. and Willam, K. (1994). Finite element analysis of elastoplastic discontinuities. Journal of Engineering Mechanics 120, 2428-2442.

[25] Iordache, M. M. (1996). Failure analysis of classical and micropolar elastoplastic materials. report CU/SR-96/2. Department of Civil, Enviromental and Archetectural Engineering, University of Colorado.

[26] Iordache, M.M. and Willam, K. (1998). Localized failure analysis in elastoplastic Cosserat continua. Comp. Meth. Appl. Mech. Engrg. 151, 559-586.

[27] Oliver, J., Cervera, M. and Manzoli, O. (1998). On the use of strain-softening models for the simulation of strong discontinuities in solids. Material instabilities in solids, 107-123, Wiley.

[28] Oliver, J., Cervera, M. and Manzoli, O. (1999). Strong discontinuities and continuum plasticity models: the strong discontinuity approach. Int. J. of Plasticity, 15, 319-351.

[29] Cervera, M., Chiumenti, M., Di Capua, D. (2012). Benchmarking on bifurcation and localization in J2 plasticity for plane stress and plane strain conditions. Computer Methods in Applied Mechanics and Engineering, 241-244, 206-224.

[30] Wu, J.Y. and Cervera, M. (2014). Thermodynamically consistent plastic-damage framework for localized failure: material model and strain localization analysis. Submitted to Int. J. of Plasticity.

[31] Cervera, M., Agelet de Saracibar, C. and Chiumenti, M. (2002). COMET: COupled MEchanical and Thermal analysis. Data Input Manual, Version 5.0, Technical report IT-308. Available from: <http://www.cimne.upc.es>.

[32] COMET: Coupled Mechanical and Thermal analysis $<$ http://www.cimne.com/comet/>.

[33] GiD: The Personal Pre and Post Processor (2009). <http://www.gidhome.com>. 